\title{
SOME DEGENERACY THEOREMS FOR ENTIRE FUNCTIONS WITH VALUES IN AN ALGEBRAIC VARIETY $\left({ }^{1}\right)$
}

\author{
BY \\ JAMES A. CARLSON
}

\begin{abstract}
In the first part of this paper we prove the following extension theorem. Let $P_{q}^{*}$ be a $q$-dimensional punctured polycylinder, i.e. a product of disks and punctured disks. Let $W_{n}$ be a compact complex manifold such that the bundle of holomorphic $q$-forms is positive in the sense of Grauert. Let $f: P_{q}^{*} \rightarrow W_{n}$ be a holomorphic map whose Jacobian determinant does not vanish identically. Then $f$ extends as a rational map to the full polycylinder $P_{q}$. In the second half of the paper we prove the following generalization of the little Picard theorem to several complex variables: Let $V \subset \boldsymbol{P}_{n}$ be a hypersurface of degree $d \geqq n+3$ whose singularities are locally normal crossings. Then any holomorphic map $f: C^{n} \rightarrow \boldsymbol{P}_{n}-V$ has identically vanishing Jacobian determinant.
\end{abstract}

0. Introduction. The purpose of this paper is twofold. First, we prove an extension theorem for holomorphic maps into a compact complex manifold $W_{n}$ such that $\wedge^{q} T_{W_{n}}^{*}$ is positive in the sense of Grauert. Second, we discuss a generalization of the little Picard theorem in several complex variables. Thus, in the first section we apply the Ahlfors-Chern-Kobayashi version of the Schwarz lemma to show

THEOREM A. Let $P_{q}^{*}=\left(\Delta^{*}\right)^{k} \times \Delta^{q-k}$ be a punctured polycylinder, and let $W_{n}$ be a compact complex manifold such that $\wedge^{q} T_{W_{n}}^{*}$ is positive in the sense of Grauert. Then any nondegenerate map $f: P_{q}^{*} \rightarrow W_{n}$ extends to a rational map $f^{\prime}: P_{q} \rightarrow W_{n}$. If $\wedge^{q} T_{W_{n}}^{*}$ is very ample, then $f^{\prime}$ is actually holomorphic.

We say that a map is rational on $P_{q}$ if it has no essential singularities along any of the branches $z_{1}=0, \ldots, z_{q}=0$. From this we obtain

COROllary. Let $\wedge^{q} T_{W_{n}}^{*}$ be positive in the sense of Grauert. Then any holomorphic map $f: C^{q} \rightarrow W_{n}$ is totally degenerate, i.e., the Jacobian determinant $|J(f)|$ vanishes identically.

The above theorem was first proved by Griffiths [3] in the equidimensional case when $\bigwedge^{n} T_{W_{n}}^{*}=K_{W}$ is very ample $\left({ }^{2}\right)$. The more general case, for $q \neq n$, is based on

Received by the editors May 25, 1971 and, in revised form, August 31, 1971.

AMS 1970 subject classifications. Primary 32D20, 32H25.

Key words and phrases. Several complex variables, extension of holomorphic maps, generalization of Picard theorem, Schottky-Landau theorem, branched cover.

${ }^{(1)}$ This work was supported by an NSF Graduate Fellowship.

$\left({ }^{2}\right)$ A proof for $K_{W}$ positive has recently been given by S. Kobayashi.

Copyright (C) 1972, American Mathematical Society 
the proof in [3] plus simple curvature arguments. It should be remarked that Griffiths' equidimensional theorem is the most important for the applications, for example, to Picard-type problems in several complex variables.

In the second section we give a simplified proof of a fundamental Nevanlinna estimate of Kodaira. Kodaira's estimate deals with holomorphic mappings of the $n$-ball of radius $R$ in $C^{n}$ into $W_{n}$, where $W_{n}$ is a complex manifold of general type (see $\$ 2$ for definitions). A consequence of this estimate is that if $f: B_{n}(R) \rightarrow W_{n}$ is normalized so that $f(0)=p_{0}$ and $|J f(0)|=1$, then there is an absolute constant $R_{\max }\left(n, p_{0}\right)$ such that $R \leqq R_{\max }$. This is a generalization of the classical SchottkyLandau theorem.

In the third section we apply the theorems of Griffiths and Kodaira to prove the following generalization of the little Picard theorem:

THEOREM B. Let $f: \boldsymbol{C}^{n} \rightarrow \boldsymbol{P}_{n}-V$ be holomorphic, and suppose that $V$ is a hypersurface of degree $d \geqq n+3$ whose singularities are locally normal crossings. Then $f$ is totally degenerate.

Thus, if $n=1$, this says that $f: C \rightarrow \boldsymbol{P}_{1}-\{0,1, a, \infty\}$ is constant, a crude version of Picard's theorem. Two interesting special cases of the above theorem occur when $V$ is smooth and when $V$ is the union of smooth hypersurfaces $V_{1}, \ldots, V_{k}$ meeting transversely and such that no more than $n$ components pass through any point. For example, if the $V_{i}$ are hyperplanes, we conclude that any holomorphic map of $\boldsymbol{C}^{n}$ into $\boldsymbol{P}_{n}-\left(V_{1} \cup \cdots \cup V_{n+3}\right)$ must be totally degenerate. This gives a partial answer to a problem of Chern, who asked the same question for $n+2$ hyperplanes $\left({ }^{3}\right)$. We should remark that the more obvious generalization of Picard's theorem, namely, that $f: \boldsymbol{C}^{n} \rightarrow \boldsymbol{P}_{n}-V$ be constant, is false in the generality of the above theorem. In fact, we have the following instructive example of Peter Kiernan.

EXAMPLE. Let $F_{d} \subset \boldsymbol{P}_{n}$ be the Fermat hypersurface of degree $d$ defined by the homogenous equation $z_{0}^{d}+\cdots+z_{n}^{d}=0$. Set $p=$ greatest integer in $n / 2$. Then there is a nondegenerate holomorphic map $f: \boldsymbol{C}^{p} \rightarrow \boldsymbol{P}_{n}-F_{d}$ for any $d$. To see this, consider the affine equation for $F_{d}: 1+w_{1}^{d}+\cdots+w_{n}^{d}=0$. We define $f$ for $n$ even by $f\left(\mu_{1}, \ldots, \mu_{p}\right)=\left(\mu_{1}, \varepsilon_{1} \mu_{1}, \mu_{2}, \varepsilon_{2} \mu_{2}, \ldots, \mu_{p}, \varepsilon_{p} \mu_{p}\right)$ where the $\varepsilon_{i}$ are $d$ th roots of -1 .

What Kiernan's example shows here is that for more refined versions of Picard's theorem one must do more than require $V$ to have high degree.

In the fourth section we prove a version of the big Picard theorem in several complex variables under the assumption that $V$ be smooth and have high degree. This is applied to give the following rigidity theorem:

Proposition. Let $V_{1}$ and $V_{2}$ be nonsingular hypersurfaces of degree $d \geqq n+3$ in $\boldsymbol{P}_{n}$. Then $\boldsymbol{P}_{n}-V_{1}$ and $\boldsymbol{P}_{n}-V_{2}$ are biholomorphic if and only if there is a projective linear automorphism $\beta$ which carries $V_{1}$ onto $V_{2}$. Moreover, the complex automorphism group of $\boldsymbol{P}_{n}-V_{1}$ is finite.

$\left.{ }^{3}\right)$ Chern's conjecture has been answered by recent work of Mark Green. 
It is my pleasure to acknowledge the many helpful conversations with Phillip Griffiths which made this work possible.

I. Intermediate volume forms and the Schwarz lemma. The aim of this section is to formulate and prove an extension theorem for holomorphic mappings of a $q$-dimensional punctured polycylinder into an $n$-dimensional complex manifold, where $q \leqq n$. The first step is to prove a generalization of the Ahlfors comparison theorem for volume elements on the unit disk. The proof is a rephrasing of the one found in [3]. Recall that if $P_{q}(R)=P(R)$ is the $q$-dimensional polycylinder $\left\{z|| z_{i} \mid<R, i=1, \ldots, q\right\}$, then there is a volume element invariant under all complex automorphisms of $P(R)$ given by

$$
\mu_{R}=i^{q^{2}+q} a^{q} R^{2 q} \frac{d z_{1} \wedge \cdots \wedge d z_{q} \wedge d \bar{z}_{1} \wedge \cdots \wedge d \bar{z}_{q}}{\prod_{j=1}^{q}\left(R^{2}-\left|z_{j}\right|^{2}\right)^{2}}
$$

Let $\nu=i^{q^{2}+q} g d z_{1} \wedge \cdots \wedge d z_{q} \wedge d \bar{z}_{1} \wedge \cdots \wedge d \bar{z}_{q}$ be a pseudovolume element on $P(R)$, i.e., $g>0$ except on an analytic subset $\mathscr{D}$. Now the Ricci form of a volume element $\nu$ given locally by $i^{q^{2}+q} h d z_{1} \wedge \cdots \wedge d z_{q} \wedge d \bar{z}_{1} \wedge \cdots \wedge d \bar{z}_{q}$ on an arbitrary complex manifold $W_{q}$ is given by

$$
\operatorname{Ric}(\nu)=d d^{c} \log h=2 i \partial \bar{\partial} \log h .
$$

Thus Ric $(\nu)$ is a real nonnegative $(1,1)$ form. If $\nu$ is a pseudovolume element, we will define the Ricci form on $P(R)-\mathscr{D}$. We want to prove the following lemma:

Lemma 1 (Ahlfors, Chern, Kobayashi). Let $v$ be a pseudovolume element on $P_{q}(R)$ such that $\operatorname{Ric}(v)$ is positive definite and $\operatorname{Ric}(\nu)^{q} \geqq c_{0} \nu$ on $P(R)-\mathscr{D}$. Then the normalization constant $a$ in (1) can be chosen such that $\nu \leqq \mu_{R}$ on $P(R)$.

Proof. (i) We first recall that with respect to $\mu_{R}, P$ is Einstein-Kähler, i.e., there is a constant $c_{1}$ such that

$$
\operatorname{Ric}(\mu)^{q}=c_{1} \mu .
$$

To see this, note that the group $G$ of complex automorphisms generated by Möbius transformations in each of the variables $z_{j}$ together with the maps $z_{i} \rightarrow z_{j}$ leaves $\mu$ invariant. Moreover, $G$ is transitive on $P$, so that if (3) holds at one point $x_{0} \in P$, then it holds for all $x \in P$.

(ii) Write $\nu=h_{\rho} \mu_{\rho}$ on $P(\rho)$. Thus we have

$$
h_{\rho}=\frac{g \prod_{j=1}^{q}\left(\rho^{2}-\left|z_{j}\right|^{2}\right)^{2}}{a^{q} \rho^{2 q}} .
$$

We must prove $h_{R} \leqq c$ for some constant $c$. Note that it is sufficient to prove $h_{\rho} \leqq c$ for $\rho<R$, since $h_{\rho} \rightarrow h_{R}$ as $\rho \rightarrow R$. For $\rho<R, h_{\rho}$ is either identically zero or has a positive interior maximum. This follows from the fact that $g$ is continuous and bounded on $P(\rho)$, so that $h_{\rho}(w) \rightarrow 0$ as $w \rightarrow \partial P(\rho)$. There is nothing to prove in 
the first case, so we assume $h_{\rho}$ has a positive maximum at $x_{\rho}$. Note that $g\left(x_{\rho}\right)>0$ if $h_{\rho}\left(x_{\rho}\right)>0$, so that $x_{\rho} \in P(\rho)-\mathscr{D}$. Thus we have

$$
0 \geqq d d^{c} \log h_{\rho}\left(x_{\rho}\right)=\operatorname{Ric} \nu\left(x_{\rho}\right)-\operatorname{Ric} \mu_{\rho}\left(x_{\rho}\right),
$$

or

$$
\operatorname{Ric} \mu_{\rho}\left(x_{\rho}\right) \geqq \operatorname{Ric} \nu\left(x_{\rho}\right) .
$$

Using the fact that $P$ is Einstein-Kähler together with $\operatorname{Ric} \nu^{q} \geqq c_{0} \nu$, we obtain

$$
c_{1} \mu_{\rho}\left(x_{\rho}\right) \geqq c_{0} \nu\left(x_{\rho}\right) .
$$

This shows that $h_{\rho}(x) \leqq h_{\rho}\left(x_{\rho}\right) \leqq c_{1} / c_{0}=c$, as desired.

To state the next result, we recall a few standard facts about curvature. Let $h$ be a hermitian metric in a complex vector bundle $E$ on $V$, and let $\theta(h)$ be the curvature form. Thus $\theta$ is a section of $\operatorname{Hom}(E, E) \otimes T_{V}^{*} \otimes \bar{T}_{V}^{*}$, where $T_{V}$ is the holomorphic tangent bundle of $V$. Now choose an orthonormal frame field $\left\{e_{\sigma}\right\}$ of $E$ near $x \in V$ and let $\theta_{\sigma}^{\rho}$ be the curvature form of $E$ relative to $\left\{e_{\sigma}\right\}$. Thus $\theta$ is given locally by

$$
\theta=\sum \theta_{o i j}^{o} d z_{i} \wedge d \bar{z}_{j}
$$

and we have $\theta_{\sigma i j}^{o}=\bar{\theta}_{o i j}^{\sigma}$. Hence

$$
\theta(\xi \otimes \eta)=\sum \theta_{\sigma 1}^{o} \xi^{\rho} \xi^{\sigma} \eta^{i} \bar{\eta}^{j}
$$

defines a quadratic form, where $\xi \in E_{x}$ and $\eta \in T_{V, x}$ (see [2, p. 201]). We say that $E$ is negative if $\theta(\xi \otimes \eta)$ is everywhere negative definite. Now let $V_{n}$ be $n$ dimensional, and suppose henceforth that there is a metric $\nu$ on $\wedge^{q} T_{V}$ such that $\theta(\nu)$ is negative. Note that a volume form on $V_{q}$, given locally by $\nu=i^{q^{2}+q} g d z_{1} \wedge$ $\cdots \wedge d z_{q} \wedge d \bar{z}_{1} \wedge \cdots \wedge d \bar{z}_{q}$, is naturally a metric on $\wedge^{q} T_{V}=K_{V}^{*}$. Thus we call such a $\nu$ on $V_{n}$ an intermediate volume form, or a $q$-volume.

In order to prove the Schwarz lemma for a $q$-volume, we need a generalization of the Ricci form. To give this, let $\Sigma_{V}$ be the sphere bundle in $\wedge^{q} T_{V}$ defined by the metric $\nu$, and let $\pi: \Sigma_{V} \rightarrow V$ be the projection. Let $\xi$ be the canonical section of length one of the bundle $\pi^{*} \wedge^{q} T_{V}$ on $\Sigma_{V}$. The generalized Ricci form of $\nu$ is a real, positive definite section of $\pi^{*}\left(T_{V} \otimes \bar{T}_{V}\right)$ which is defined locally by

$$
\operatorname{Ric}^{\#}(\nu)=-i \sum \theta_{\sigma i j}^{\rho} \xi^{\rho} \xi^{\sigma} d z_{i} \wedge d \bar{z}_{j}
$$

where $\theta_{\sigma}^{\rho}$ is the curvature form of $\bigwedge^{q} T_{V}$ relative to an orthonormal frame $\left\{e_{\sigma}\right\}$ near $p \in V$.

LEMMA 2. Let $W \subseteq V$ be a complex submanifold. Hence $\nu_{V}$ restricts to give a metric $\nu_{W}$ in $\bigwedge^{a} T_{W}$. Then $\Sigma_{w} \subseteq \Sigma_{V}$ and $\operatorname{Ric}^{\#}\left(\nu_{W}\right) \geqq \operatorname{Ric}^{\#}\left(\nu_{V}\right)$ on $\Sigma_{W}$.

Proof. $\wedge^{q} T_{W}$ is a subbundle of $\left.\wedge^{q} T_{V}\right|_{w}$. The usual argument (see [2, pp. 196-201]) with the second fundamental form shows that curvature decreases in the 
subbundle: $\theta\left(\nu_{W}\right)(\xi \otimes \eta) \leqq \theta\left(\nu_{V}\right)(\xi \otimes \eta)$ where $\xi \in \wedge^{q} T_{W, x}$ and $\eta \in T_{W, x}$. This means that

$$
-i \sum \theta\left(\nu_{W}\right)_{\sigma i j}^{\rho} \xi^{\rho} \bar{\xi}^{\sigma} d z_{i} \wedge d \bar{z}_{j} \geqq-i \sum \theta\left(\nu_{V}\right)_{\sigma i j}^{\rho} \xi^{\rho} \bar{\xi}^{\sigma} d z_{i} \wedge d \bar{z}_{j}
$$

as desired.

REMARKS. (i) Note that if $V$ is $n$-dimensional and $\bigwedge^{n} T_{V}$ is negative relative to a volume element $\nu$, then both Ric $(\nu)$ and Ric\# $(\nu)$ are positive. For example, the unit disk with Poincaré volume

$$
\mu=(i d z \wedge d \bar{z}) /\left(1-|z|^{2}\right)^{2}
$$

has constant negative Gauss curvature -4 , whereas $\operatorname{Ric}(\mu)$ is positive. Thus negative curvature corresponds to positive Ricci form. Ric\# should be thought of as the generalization of Gauss curvature needed to generalize the Ahlfors lemma.

(ii) For $q=1$ the above lemma reduces to the statement that if $W \subseteq V$ is a submanifold, then the holomorphic sectional curvatures of $W$ are no greater than the sectional curvatures of $V$. The proof is also the same, except that we have done things with a globally defined form which is positive.

The next lemma is a generalization of the statement that if $V$ is compact and has negative sectional curvatures, then the sectional curvatures are bounded away from zero.

LEMMA 3. Let $V$ be compact, and suppose that $\wedge^{q} T_{V}$ is negative relative to $\nu$. If $\xi$ is the canonical section of length one on $\pi^{*} \wedge^{q} T_{V}$, then $\operatorname{Ric}^{\#}(\nu)^{q}(\xi) \geqq c>0$.

Proof. Note that Ric ${ }^{\#}(\nu)^{q}$ is a positive section of $\pi^{*}\left(\bigwedge^{q} T_{V}^{*} \otimes \bigwedge^{q} \bar{T}_{V}^{*}\right)$. Evaluating Ric $\#(\nu)^{q}$ on $\xi$ gives a positive function on a compact manifold, from which the result follows.

We can now reinterpret the condition Ric $(\nu)^{q} \geqq c_{0} \nu$ in Lemma 1 . Note that both Ric $(\nu)^{q}$ and $\nu$ give metrics in $K_{P^{\prime}}^{*}\left(P^{\prime}=P-\mathscr{D}\right)$, so that to verify the above inequality, it is enough to verify it on sections $\xi$ of $K_{P}^{*}$ which have length one relative to $\nu$. Thus the Ricci condition is locally $\operatorname{Ric}(\nu)^{q}(\xi) \geqq c_{0}$ for $\nu(\xi)=1$, or equivalently, $\operatorname{Ric}^{\#}(\nu)^{q}(\xi) \geqq c_{0}$ where $\xi$ is the canonical section of length one. This leads to the following result.

Proposition 1. Let $V_{n}$ be a compact complex manifold with a q-volume $\nu_{V}$ such that $\wedge^{q} T_{V}$ is negative. Let $f: P_{q}(R) \rightarrow V_{n}$ be a nondegenerate holomorphic map. Then by a suitable choice of the normalization constant in (1), we obtain $f^{*} \nu_{V} \leqq \mu_{R}$. Moreover, the choice of constant is independent of $f$.

Proof. Let $\mathscr{D}$ be the locus of $J(f)=0$ on $P(R)$. Then $f^{*} \nu_{V}$ is a pseudometric on $K_{P(R)}^{*}$ which is a metric on $K_{P^{\prime}}^{*}\left(P^{\prime}=P-\mathscr{D}\right)$. By Lemma 1, we must show Ric $\left(f^{*} \nu_{V}\right)^{q}$ $\geqq c_{0} f^{*} \nu_{V}$ on $P^{\prime}$. By the above remarks, this is equivalent to showing $\operatorname{Ric}^{\#}\left(f^{*} \nu_{V}\right)^{q}(\xi)$ $\geqq c_{0}$ on $\Sigma_{P^{\prime}}$, where $\Sigma_{P}$ is the circle bundle of $K_{P^{\prime}}^{*}$ defined by the metric $f^{*} \nu_{V}$. Now $f$ is locally a holomorphic diffeomorphism of $P^{\prime}$ onto $f\left(P^{\prime}\right)$ which gives an isomorphism $f_{*}: \bigwedge^{q} T_{P^{\prime}} \rightarrow \bigwedge^{q} T_{f\left(P^{\prime}\right)}$ preserving metrics. Here $\bigwedge^{q} T_{f\left(P^{\prime}\right)}$ is equipped 
with the metric $\nu_{f}$ gotten by restriction of $\nu_{v}$. Therefore it is enough to prove $\operatorname{Ric}^{\#}\left(\nu_{f}\right)^{q}(\xi) \geqq c_{0}$ on $\Sigma_{f\left(P^{\prime}\right)}$. From Lemma 2 we get $\operatorname{Ric}^{\#}\left(\nu_{f}\right)(\xi) \geqq \operatorname{Ric}^{\#}\left(\nu_{V}\right)(\xi)$ in $\pi^{*}\left(T_{f\left(P^{\prime}\right)}^{*} \otimes \bar{T}_{f\left(P^{\prime}\right)}^{*}\right)$. These are positive sections, hence $\operatorname{Ric}^{\#}\left(\nu_{f}\right)^{q} \geqq \operatorname{Ric}^{\#}\left(\nu_{V}\right)^{q}(\xi)$ in $\bigwedge^{q} T_{f\left(P^{\prime}\right)}^{*} \otimes \bigwedge^{q} \bar{T}_{f\left(P^{\prime}\right)}^{*}$. Now Lemma 3 gives $\operatorname{Ric}^{\#}\left(\nu_{V}\right)^{q}(\xi) \geqq c_{0}$ everywhere, hence $\operatorname{Ric}^{\#}\left(\nu_{f}\right)^{q}(\xi) \geqq c_{0}$, as desired.

REMARKS. This argument specializes to give the usual result on distancedecreasing for negative sectional curvatures when $q=1$. When $q=n$, it specializes to Griffiths' Schwarz lemma in [3]. The proof in the general case is by interpolation.

To state the next result, suppose that $V$ is an $n$-dimensional complex manifold with a metric $\nu_{V}$ in $S^{l} \wedge^{q} T_{V}$, where $S^{l} E=S^{l}(E)$ denotes the $l$ th symmetric power of the bundle $E$. Let $f: W \rightarrow V$ be a holomorphic map, where $W$ is $q$-dimensional. Then $f^{*} \nu_{V}$ is a metric on $S^{l} \wedge^{q} T_{W}$ which has the local form

$$
g\left(i^{q^{2}+q} d z_{1} \wedge \cdots \wedge d z_{q} \wedge d \bar{z}_{1} \wedge \cdots \wedge d \bar{z}_{q}\right)^{l} .
$$

This defines a volume form $\left(f^{*} \nu_{V}\right)^{1 / l}$ which has the local form

$$
i^{q^{2}+q} g^{1 / l} d z_{1} \wedge \cdots \wedge d z_{q} \wedge d \bar{z}_{1} \wedge \cdots \wedge d \bar{z}_{q} .
$$

Proposition 2. Let $V_{n}$ be a compact complex manifold such that $S^{l} \wedge^{a} T_{V}$ is negative relative to a metric $\nu_{V}$. Let $f: P_{q}(R) \rightarrow V$ be nondegenerate and holomorphic. Then the normalization constant in (1) can be chosen independently of $f$ such that $\left(f^{*} \nu_{V}\right)^{1 / l} \leqq \mu_{R}$.

Proof. We want to mimic the proof of Proposition 1. First note that $\nu_{V}$ defines a Ricci form by the same formula (4) as before, except that the Ricci form is now defined on $\pi^{*}\left(T_{V}^{*} \otimes \bar{T}_{V}^{*}\right)$, where $\pi: \Sigma_{V} \rightarrow V$ is the projection from the sphere bundle of $S^{l} \wedge^{q} T_{V}$. By the same arguments which establish Lemmas 2 and 3, we get

LEMma 2'. Let $W \subseteq V$ be a complex submanifold. Hence $\nu_{V}$ restricts to give a metric $\nu_{W}$ in $S^{l} \wedge^{q} T_{W}$. Then $\Sigma_{W} \subseteq \Sigma_{V}$ and $\operatorname{Ric}^{\#}\left(\nu_{W}\right) \geqq \operatorname{Ric} \#\left(\nu_{V}\right)$ on $\Sigma_{W}$.

Lemma 3'. Let $V$ be compact, and suppose that $S^{l} \bigwedge^{q} T_{V}$ is negative relative to $\nu$. If $\xi$ is the canonical section of length one of $\pi^{*} S^{l} \wedge^{q} T_{V}$, then $\left[\operatorname{Ric}(\nu)^{q}\right]^{l}(\xi) \geqq c>0$.

Now let $f^{*} \nu_{V}=g\left(i^{q^{2}+q} d z_{1} \wedge \cdots \wedge d \bar{z}_{q}\right)^{l}$ so that $\left(f^{*} \nu_{V}\right)^{1 / l}=i^{q^{2}+q} g^{1 / l} d z_{1} \wedge \cdots \wedge d \bar{z}_{q}$. To apply Lemma 1 to $\left(f^{*} \nu_{V}\right)^{1 / l}$ and thus to complete the proof, we must show

(a) $\operatorname{Ric}\left(f^{*} \nu_{V}\right)^{1 / l}$ is positive definite,

(b) $\left(\operatorname{Ric}\left(f^{*} \nu_{V}\right)^{1 / l}\right)^{q} \geqq c_{0}\left(f^{*} \nu_{V}\right)^{1 / l}$.

Proof of (a). Note that (a) follows if Ric $\left(\nu_{f}\right)^{1 / l}$ is positive definite on $f\left(P^{\prime}\right)$,

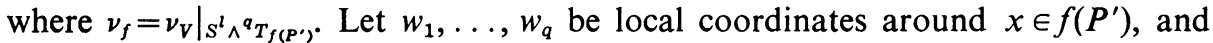
set $\nu_{f}=h\left(i^{q^{2}+q} d z_{1} \wedge \cdots \wedge d \bar{z}_{q}\right)^{l}$. If $\xi$ and $\xi^{\prime}$ are the canonical sections of the sphere bundles $\Sigma\left(S^{l} \bigwedge^{q} T_{f\left(P^{\prime}\right)}\right)$ and $\Sigma\left(\bigwedge^{q} T_{f\left(P^{\prime}\right)}\right)$, then we have

$$
\operatorname{Ric}^{\#}\left(\nu_{f}\right)(\xi)=d d^{c} \log h=l \cdot d d^{c} \log h^{1 / l}=l \cdot \operatorname{Ric}^{\#}\left(\nu_{f}^{1 / l}\right)\left(\xi^{\prime}\right) .
$$


By Lemma $2^{\prime}$, Ric\# $\left(v_{f}\right)$ is positive definite, from which we conclude that $\operatorname{Ric}^{\#}\left(f^{*} \nu_{V}\right)^{1 / l}$ is positive definite.

Proof of (b). We claim that (b) follows from

(c) $\left[\left(\operatorname{Ric} f^{*} \nu_{V}\right)^{q}\right]^{l} \geqq c_{1} f^{*} \nu_{V}$.

Note that (c) holds because of Lemma $3^{\prime}$. Expressing (b) and (c) in local coordinates we have

$$
\begin{aligned}
& \left(d d^{c} \log g^{1 / l}\right)^{q} \geqq i^{q^{2}+q} c_{0} g^{1 / l} d z_{1} \wedge \cdots \wedge d \bar{z}_{q}, \\
& {\left[\left(d d^{c} \log g\right)^{q}\right]^{l} \geqq c_{1} g\left[i^{q^{2}+q} d z_{1} \wedge \cdots \wedge d \bar{z}_{q}\right]^{l} .}
\end{aligned}
$$

Let $d d^{c} \log g=i \sum g_{i j} d z_{i} \wedge d \bar{z}_{j}$, so that $\left(d d^{c} \log g\right)^{q}=\operatorname{det}\left(g_{i j}\right)\left(i^{q^{2}+q} d z_{1} \wedge \cdots \wedge d \bar{z}_{q}\right)$. Hence $\left(b^{\prime}\right)$ and $\left(c^{\prime}\right)$ can be written as

$$
\begin{gathered}
(1 / l)^{q} \operatorname{det}\left(g_{i j}\right) \geqq c_{0} g^{1 / l}, \\
\left(\operatorname{det}\left(g_{i j}\right)\right)^{l} \geqq c_{1} g .
\end{gathered}
$$

Since Ric $\left(_{f}\right)$ is positive, we know that $\left(g_{i j}\right)$ is a positive definite matrix. It is now clear that $\left(\mathrm{b}^{\prime \prime}\right)$ follows from $\left(\mathrm{c}^{\prime \prime}\right)$ with $c_{0}=(1 / l)^{q} c_{1}$.

To state the next result, we recall that a complex vector bundle $E$ is negative in the sense of Grauert if the zero section can be blown down to a point (see [5]). This is equivalent to the existence of a plurisubharmonic function on $E$ which is strictly plurisubharmonic on the complement of the zero section. A complex vector bundle $E$ is positive in the sense of Grauert if its dual is negative in the sense of Grauert. We recall that if $E$ is Graueri-positive then $S^{l} E$ has positive curvature for $l$ sufficiently large. We can now state the following:

Proposition 3. Let $V_{n}$ be a compact complex manifold such that $\wedge^{q} T_{V}^{*}$ is positive in the sense of Grauert. Let $f: P_{a}^{*} \rightarrow V$ be a nondegenerate holomorphic map defined on the punctured polycylinder $\left(\Delta^{*}\right)^{k} \times \Delta^{q-k}$. Then $f$ extends to a rational $\left({ }^{4}\right)$ $\operatorname{map} \bar{f}: P_{q} \rightarrow V$.

Proof. (i) Because $\bigwedge^{q} T_{V}^{*}$ is Grauert-positive, $S^{l} \bigwedge^{q} T_{V}^{*}$ has positive curvature for some $l \gg 0$ (see [5], [2]). By choosing $l$ even larger, we may assume that $S^{l} \wedge^{q} T_{V}^{*}$ is very ample (see [2]). This means that $V$ is immersed into a Grassmannian $\operatorname{Gr}(m, N)$ by the global sections of $S^{l} \wedge^{q} T_{V}^{*}$. To be explicit, we choose a basis $\omega_{1}, \ldots, \omega_{N}$ for the vector space $\Gamma$ of global sections. Evaluation of a section on the fiber gives the exact sequence

$$
0 \rightarrow F \rightarrow V \times \Gamma \rightarrow S^{l} \wedge^{q} T_{V}^{*} \rightarrow 0,
$$

which defines a map $\gamma: V \rightarrow \operatorname{Gr}(m, N)$ by sending $x$ to the subspace $F_{x}$ of $\Gamma$. We now fix a metric in $S^{l} \wedge^{q} T_{V}$ by $\nu_{V}=\omega_{1} \wedge \bar{\omega}_{1}+\cdots+\omega_{N} \wedge \bar{\omega}_{N}$.

(ii) To extend $f: P_{q}^{*} \rightarrow V_{n}$ to a rational map $\bar{f}: P_{q} \rightarrow V_{n}$, it is sufficient to extend each of the holomorphic sections $f^{*} \omega_{i}$ of $S^{l} \wedge^{q} T_{P^{\star}}^{*}$ to a rational section of

$\left.{ }^{4}\right)$ Rational means that $f$ has no essential singularities along $z_{1}=0, \ldots, z_{k}=0$. 
$S^{l} \wedge^{q} T_{P}^{*}$. This is because the $\omega_{i}$ give local coordinates on $V$. We will show that $f^{*} \omega_{i}$ has at most a pole of order $l-1$ along $z_{i}=0, i=1, \ldots, k$. Write $f^{*} \omega_{i}$ $=g_{i}\left(i^{q^{2}+q} d z_{1} \wedge \cdots \wedge d \bar{z}_{q}\right)^{l}$. If $g_{i}$ has an essential singularity or a pole of order $>N$ along some $z_{j}=0$, then there is a $c>0$ such that, in the punctured polycylinder $P^{*}\left(\frac{1}{2}\right)$ of radius $\frac{1}{2}$, we have $\left|g_{i}\right|>c /\left(z_{1} \cdots z_{k}\right)^{N}$. For if not, the Riemann extension theorem yields $g_{i}=h /\left(z_{1} \cdots z_{k}\right)^{N}$, where $h_{i}$ is holomorphic on $P\left(\frac{1}{2}\right)$, a contradiction. Suppose that we can prove

$$
\int\left(f^{*}\left(\omega_{i} \wedge \bar{\omega}_{i}\right)\right)^{1 / l}<\infty
$$

Then we have

$$
\begin{aligned}
\infty & >\int_{P^{*}}\left(f^{*}\left(\omega_{i} \wedge \bar{\omega}_{i}\right)\right)^{1 / l} \\
& =\int_{P^{*}}\left|g_{i}\right|^{2 / l} d z_{1} \wedge \cdots \wedge d \bar{z}_{q}>\lim _{\varepsilon \rightarrow 0} c^{\prime} \int_{\varepsilon}^{1 / 2} \frac{r_{1} d r_{1}}{r_{1}^{2 N / l}} \cdots \int_{\varepsilon}^{1 / 2} \frac{r_{k} d r_{k}}{r_{k}^{2 N / l}} .
\end{aligned}
$$

Hence

$$
\infty>\text { const }+\frac{\text { const }}{(2-2 N / l)^{k}} \cdot \lim _{\varepsilon \rightarrow 0}\left(\varepsilon^{2-2 N / l}\right)^{k}
$$

from which we conclude $2-2 N / l>0$, or $N<l$.

(iii) It remains to show (*). First, we show that

$$
\int_{P^{*}}\left(f^{*}\left(\omega_{i} \wedge \bar{\omega}_{i}\right)\right)^{1 / l} \leqq N\left(\frac{1}{N}\right)^{1 / l} \int_{P^{*}}\left(f^{*} \nu_{V}\right)^{1 / l}
$$

Now $f^{*} \nu_{V}=\sum_{i=1}^{N} f^{*}\left(\omega_{i} \wedge \bar{\omega}_{i}\right)$ so that

$$
f^{*} \nu_{V}=\left(\sum_{i=1}^{N}\left|g_{i}\right|^{2}\right)\left(i^{q^{2}+q} d z_{1} \wedge \cdots \wedge d \bar{z}_{q}\right)^{l} .
$$

Using the fact that the function $\chi \rightarrow \chi^{1 / l}$ is concave $(5)$, we get

$$
\frac{1}{N}\left|g_{i}\right|^{2 / l} \leqq \frac{1}{N} \sum_{i=1}^{N}\left|g_{i}\right|^{2 / l} \leqq\left(\frac{1}{N} \sum\left|g_{i}\right|^{2}\right)^{1 / l},
$$

which gives $(1 / N)\left(f^{*}\left(\omega_{i} \wedge \bar{\omega}_{i}\right)\right)^{1 / l} \leqq(1 / N)^{1 / l}\left(f^{*} \nu_{V}\right)^{1 / l}$, and hence the claim.

Now let $\kappa: P \rightarrow P^{*}$ be the universal covering map. The Poincaré volume $\mu$ on $P$ is invariant under covering transformations, so it descends to give a volume form $\mu_{P *}$ on $P^{*}$. Therefore, $\left(f^{*} \nu_{V}\right)^{1 / l} \leqq \mu_{P *}$ on $P^{*}$ is equivalent to $\left(\kappa^{*} f^{*} \nu_{V}\right)^{1 / l} \leqq \mu_{P}$ on $P$. The latter statement follows from the Schwarz lemma applied to the function $f \circ \kappa$.

(5) If $E$ is a measure space such that $\mu(E)=1$, and $\psi$ is concave, then Jensen's inequality says $\int_{E} \psi \circ f d \mu=\psi\left(\int_{E} f d \mu\right)($ see $[10$, p. 61$])$. 
Now the volume of the concentric polycylinder $P\left(\frac{1}{2}\right)$ is finite in the Poincaré volume, which implies that $\mu_{P^{*}}\left(P^{*}\left(\frac{1}{2}\right)\right)<\infty$. Therefore we conclude that

$$
\begin{aligned}
\int_{P^{*}(1 / 2)}\left(f^{*}\left(\omega_{i} \wedge \bar{\omega}_{i}\right)\right)^{1 / l} & \leqq N\left(\frac{1}{N}\right)^{1 / l} \int_{P^{*}(1 / 2)}\left(f^{*} \nu_{V}\right)^{1 / l} \\
& \leqq N\left(\frac{1}{N}\right)^{1 / l} \int_{P^{*}(1 / 2)} \mu_{P^{*}}<\infty
\end{aligned}
$$

as desired.

REMARK. Note that if $l=1$, i.e. if $\bigwedge^{q} T_{V}^{*}$ is very ample, then $f$ extends as a holomorphic mapping. For $q=n$ this is Griffiths' extension theorem for canonical algebraic varieties [3]. The proof also establishes Griffiths' theorem when $K_{V}$ is positive.

Before giving the applications of this theorem, we must recall the notion of smooth compactifications of algebraic varieties (see [2]). Let $U$ be a smooth projective Zariski open set, i.e. $U=V-W$, where $V$ and $W$ are projective varieties. A smooth compactification of $U$ is a nonsingular projective variety $U^{\prime}$ such that $U \subseteq U^{\prime}$ is a dense open set and $U^{\prime}-U=D$ is a union of nonsingular divisors $D_{i}$ with normal crossings. Thus a local analytic equation for $D$ in $U^{\prime}$ is $z_{1}, \ldots, z_{k}=0$. This has the useful consequence that if $P$ is a polycylinder in $U^{\prime}$, then $U \cap P$ $=\left(U^{\prime}-D\right) \cap P$ is just a punctured polycylinder $\left(\Delta^{*}\right)^{k} \times \Delta^{n-k}$. Recall that $U^{\prime}$ is obtained from $V$ by quadratic transformations. Thus there is a rational holomorphic map $U^{\prime} \stackrel{\pi}{\rightarrow} V$ which is biholomorphic on $U$. Smooth compactifications are not unique. Given two compactifications $U^{\prime}, U^{\prime \prime}$, there is always a third one $U^{\prime \prime \prime}$ with rational holomorphic maps $\pi_{1}: U^{\prime \prime \prime} \rightarrow U^{\prime}$ and $\pi_{2}: U^{\prime \prime \prime} \rightarrow U^{\prime \prime}$. If $f: X \rightarrow Y$ is a rational map between algebraic varieties, then there is a subvariety $B \subseteq X$ along which $f$ is indeterminate. However, we can always find a smooth compactification $X^{\prime}$ of $X-B$ such that $\pi: X^{\prime} \rightarrow X$ pulls $f$ back to an everywhere defined function, i.e. a rational holomorphic function.

As an example, we note that $\boldsymbol{P}_{n}$ is a smooth compactification of $\boldsymbol{C}_{n}$, where $D=\boldsymbol{P}_{n-1}$ is the hyperplane at infinity. Let $z_{0}, z_{1}, z_{2}$ be homogeneous coordinates on $\boldsymbol{P}_{2}$ such that $D=\left\{z_{0}=0\right\}$. Then $z_{1} / z_{0}$ is holomorphic on $\boldsymbol{C}^{2}=\boldsymbol{P}_{2}-D$ and has a pole along $D$. However, $z_{1} / z_{0}$ is indeterminate at $[0,0,1]$ (homogeneous coordinates). To remedy this, we blow up $[0,0,1]$ to obtain a rational variety $\boldsymbol{P}_{2}^{\prime}$ and a holomorphic map $\pi: \boldsymbol{P}_{2}^{\prime} \rightarrow \boldsymbol{P}_{2}$. If $\mathscr{E}=\pi^{-1}[0,0,1]$ is the exceptional set, then $\pi$ is biholomorphic on $\boldsymbol{P}_{2}^{\prime}-\mathscr{E}$. Now $z_{1} / z_{0}$ extends from $\boldsymbol{P}_{2}^{\prime}-\mathscr{E}$ to $\boldsymbol{P}_{2}$ as an everywhere defined rational function $f: \boldsymbol{P}_{2}^{\prime} \rightarrow \boldsymbol{P}_{1}$.

An immediate corollary of Proposition 3 and the above remarks is the following extension theorem.

Corollary 1. Let $U_{q}$ be a q-dimensional Zariski open set as above, and $f: U_{q} \rightarrow V_{n}$ a nondegenerate holomorphic map. If $\bigwedge^{q} T_{V}^{*}$ is positive in the sense of 
Grauert, then $f$ extends as a holomorphic map $f^{\prime}: U_{q}^{\prime} \rightarrow V_{n}$ for a suitable smooth compactification $U_{q}^{\prime}$.

This, in turn, gives the following degeneracy theorem:

COROLlaRY 2. Let $V_{n}$ be an n-dimensional complex manifold such that $\wedge^{q} T_{V}^{*}$ is positive in the sense of Grauert. Then any holomorphic map $f: C^{q} \rightarrow V_{n}$ is totally degenerate.

Proof. By Corollary $1, f$ extends to a holomorphic map $f^{\prime}: \boldsymbol{P}_{q}^{\prime} \rightarrow V_{n}$. We know that for $l \gg 0, S^{l} \wedge^{q} T_{V}^{*}$ is very ample. Therefore we can find a global section $\omega \in \Gamma\left(S^{l} \wedge^{q} T_{V}^{*}\right)$ such that $f^{*}\left(\omega \in \Gamma\left(K_{P_{q}^{\prime}}^{l}\right)\right.$ is nonzero. But $\Gamma\left(K_{P_{q}^{\prime}}^{l}\right) \simeq \Gamma\left(K_{P_{q}}^{l}\right)=\{0\}$, a contradiction.

REMARKS. (1) The extension theorem is also valid when $\wedge^{q} T_{V}^{*}$ is very ample and $f: U_{m} \rightarrow V_{n}$ has rank $\geqq p\left(\operatorname{dim} U_{m}=m\right)$.

(2) If $\wedge^{a} T_{V}^{*}$ is positive in the sense of Grauert, then the Schottky-Landau theorem holds. This says that if $f: P_{q}(R) \rightarrow V_{n}$ is normalized so that $f(0)=p$ and $|J(f)(0)|=1\left({ }^{6}\right)$, then there is a constant $R_{\max }$ independent of $f$ such that $R \leqq R_{\max }$. The proof follows from the Schwarz lemma in exactly the same way as for the equidimensional Schottky-Landau theorem in [3].

II. The basic Kodaira estimate. We will give a modified proof of a SchottkyLandau theorem due to Kodaira [8]. To state the theorem, we recall that an algebraic manifold is of general type if

$$
\liminf _{m \rightarrow \infty} m^{-n} \operatorname{dim} H^{0}\left(V, K^{m}\right)>0
$$

where $n=\operatorname{dim} V$ and $K$ is the canonical bundle.

Theorem (Kodaira). Let $B(R)=\left\{\left.z \in C^{n}\left|\sum\right| z_{i}\right|^{2}<R\right\}$, and let $f: B(R) \rightarrow V$ be holomorphic and nondegenerate. Suppose $f$ is normalized such that $f(0)=p$ and $|J(f)(0)|=1\left({ }^{7}\right)$. If $V$ is of general type, then there is a constant $R_{\max }$ independent of $f$ such that $R \leqq R_{\max }$.

In particular, this implies that there is no nondegenerate map $f: C^{n} \rightarrow V$, where $V$ is of general type. The usefulness of Kodaira's theorem is that the numerical criterion of general type is usually much easier to compute than curvature conditions. The main point in the proof given below is that balls are used instead of polycylinders.

Conventions. We begin by establishing some conditions regarding curvature and volume forms. This will be done to avoid complicated and irrelevant constants

$\left(^{6}\right)|J(f)(0)|$ is defined by measuring $f_{*}\left(\left(\partial / \partial z_{1}\right) \wedge \cdots \wedge\left(\partial / \partial z_{q}\right)\right)$ with the metric $\nu$ in $\wedge^{a} T_{V}^{*}$, for example.

${ }^{(7)}|J(f)(0)|$ can be defined relative to coordinates on a fixed polycylindrical neighborhood $U$ of $f(0)$. 
in the final theorems. First, we let $d^{c}=(-1)^{1 / 2}(\bar{\partial}-\partial) / 4 \pi\left({ }^{8}\right)$. This is defined so that $\int_{|z|=1} d^{c} \log |z|^{2}=1$. Let $\|z\|^{2}=\left|z_{1}\right|^{2}+\cdots+\left|z_{m}\right|^{2}$, and define $\eta=d^{c} \log \|z\|$, $\psi=d \eta=d d^{c} \log \|z\|$. If we let $\sigma=\eta \wedge \psi^{n-1}$, then the sphere of radius $r$ in $\boldsymbol{C}^{n}$ has volume 1 relative to $\sigma: \int_{S[r]} \sigma=1$. If $L$ is a line bundle given by transition functions $f_{\alpha \beta}$, then sections of $L$ are locally defined functions $s_{\alpha}$ such that $s_{\alpha}=f_{\alpha \beta} s_{\beta}$. If $\left\{a_{\alpha}\right\}$ is a collection of positive $C^{\infty}$ functions satisfying $a_{\alpha}=\left|f_{\alpha \beta}\right|^{2} a_{\beta}$, then the length of a section is measured by $|s|^{2}=\left|s_{\alpha}\right|^{2} / a_{\alpha}$. The first Chern class of $L$ is defined by $c_{1}(L)$ $=d d^{c} \log a_{\alpha}$. With these definitions one verifies that an ample bundle has positive definite first Chern class. By this, we mean that if $d d^{c} \log a_{\alpha}=\left((-1)^{1 / 2} / 4 \pi\right) \sum g_{i j}^{\alpha} d z_{i} \wedge d \bar{z}_{j}$, then the hermitian matrix $\left(g_{i j}^{\alpha}\right)$ is positive definite. For example, one easily checks that the hyperplane bundle on $\boldsymbol{P}_{n}$ is positive according to this definition $\left({ }^{9}\right)$.

Proof of the basic estimates. We begin by defining a volume form on $V$. Let $L$ be the hyperplane line bundle on $V$ determined by some projective imbedding. If $V$ is of general type, then there is a section $s \in H^{0}\left(V, K^{m} \otimes L^{*}\right)$ for some $m>0\left({ }^{10}\right)$. Let $a$ be the metric on $L$ defined above. Thus $a$ is a positive $C^{\infty}$ section of $L \otimes L$. Now $s \otimes \bar{s}$ is a real nonnegative $C^{\infty}$ section of $K_{V}^{m} \otimes \bar{K}_{V}^{m} \otimes L^{*} \otimes \bar{L}^{*}$, so that $a s \otimes \bar{s}$ is a nonnegative section of $K_{V}^{m} \otimes \bar{K}_{V}^{m}$. Therefore it has a globally defined $m$ th root

$$
\Omega=(a s \otimes \bar{s})^{1 / m} \in \Gamma\left(K_{V} \otimes \bar{K}_{V}\right),
$$

which is the volume element we want. If $s$ is defined locally by $s_{\alpha}$, then the $(n, n)$ form $\Omega$ is defined locally by $\Omega_{\alpha}=a_{\alpha}^{1 / m}\left|s_{\alpha}\right|^{2 / m}$.

Now suppose that $f: B(R) \rightarrow V$ is holomorphic and nondegenerate. We will also suppose that $f(0)=p \notin(s)$, where $(s)=\{x \in V \mid s(x)=0\}$. Let $\xi$ be the comparison function defined by $f^{*} \Omega=(-1)^{n / 2} \xi d z_{1} \wedge d \bar{z}_{1} \wedge \cdots \wedge d z_{n} \wedge d \bar{z}_{n}$. Thus

$$
\xi=\left(a_{\alpha}^{1 / m} \circ f\right)\left|s_{\alpha} \circ f\right|^{2 / m}|J(f)|^{2} .
$$

Since $f$ is normalized so that $f(0)=p$ and $|J(f)(0)|=1$, we can multiply $\Omega$ by a constant independent of $f$ so that $\xi(0)=1$. The idea of Kodaira's result is to apply the proof of Jensen's theorem to $\xi$. We define two order functions by

$$
T(r)=\int_{B[r]} \xi \beta^{n}, \quad T^{\#}(r)=\int_{B[r]} \xi^{1 / n} \beta^{n},
$$

where $\beta=\left((-1)^{1 / 2} / 4 \pi\right) \sum_{j=1}^{n} d z_{j} \wedge d \bar{z}_{j}, B[r]=\left\{z \in C^{n} \mid\|z\|<r\right\}$ and $r<R$. The order function $T(r)$ has the natural interpretation as the volume of the image $f(B[r])$.

$\left.{ }^{8}\right)$ This is different from the $d^{c}$ used in $\S 1$.

( $\left.{ }^{9}\right)$ If $L$ is very ample, take a basis $s^{0}, \ldots, s^{N}$ for the global sections, and define $a_{\alpha}=\left|s_{\alpha}^{0}\right|^{2}$ $+\cdots+\left|s_{\alpha}^{N}\right|^{2}$. Since $L$ is very ample, $a$ has the form $1+\left|z_{1}\right|^{2}+\left|z_{n}\right|^{2}+$ higher order terms. Here the $z_{\mathrm{i}}$ are coordinates centered at an arbitrary point $x$. Then $d d^{c} \log a_{\alpha}$ is positive definite at $x$ (see [2]).

$\left(^{10}\right)$ We recall Kodaira's proof: Since $L=[X]$, where $X$ is a nonsingular hyperplane section of $V$, we have the exact sequence $0 \rightarrow H^{\circ}\left(V, K^{m} \otimes L^{*}\right) \rightarrow H^{\circ}\left(V, K^{m}\right) \rightarrow H^{\circ}\left(X, K_{X}^{m}\right) \cdots$. Now $\operatorname{dim} H^{\circ}\left(V, K^{m}\right)=O\left(m^{n}\right)$ whereas $\operatorname{dim} H^{0}\left(X, K_{X}^{m}\right)=O\left(m^{n-1}\right)$, so that $\operatorname{dim} H^{\circ}\left(V, K^{m} \otimes L^{*}\right)>0$ for sufficiently large $m$. 
Unfortunately, $T^{\#}(r)$ is required for technical reasons. Counting functions are defined by

$$
n(t)=\int_{Z[t]} \psi^{n-1}, \quad N(t)=\int_{0}^{t} n(s) \frac{d s}{s},
$$

where $Z[t]=f^{-1}((s)) \cap B[t]$,

$$
n_{1}(t)=\int_{R[t]} \psi^{n-1}, \quad N_{1}(t)=\int_{0}^{t} n_{1}(s) \frac{d s}{s},
$$

where $R[t]=(J) \cap B[t]$ and $(J)$ is the divisor of $J(f)=0$. The normalization conditions are simply that neither $Z=f^{-1}((s))$ nor $(J)$ pass through the origin so that both $n(t)$ and $n_{1}(t)$ converge. The functions $n(t)$ and $n_{1}(t)$ should be thought of as the area of $Z[t], R[t]$, respectively. Hence $n(t)$ and $n_{1}(t)$ are zero for small values of $t$, so the improper integrals $N(t)$ and $N_{1}(t)$ converge. We can now state

PRoposition 1. Under the assumptions of Theorem 1 we have $T(r) \geqq r^{2 n}$ and $T^{\#}(r) \geqq l_{0} r^{2 n}$, where $l_{0}$ is an absolute constant depending only on $n=\operatorname{dim} V$.

Proposition 2. Under the assumptions of Theorem 1 we have

$$
\frac{n k^{1 / n}}{m} \int_{0}^{r} T^{\#}(r) \frac{d t}{t^{2 n-1}}+\frac{1}{m} N(r)+N_{1}(r) \leqq n \log \left[\frac{1}{r^{2 n-1}} \frac{d T^{\#}}{d r}\right]+l_{1} .
$$

Here $k$ is a positive constant such that $c_{1}(L)^{n} \geqq k\left(n ! /(4 \pi)^{n}\right) \Omega$, and $l_{1}$ is an absolute constant depending only on $n$.

The Schottky-Landau theorem will follow easily from Propositions 1 and 2.

Preliminaries to the proof of both propositions. Consider $I(t)=\int_{B[t]} d d^{c} \log \xi \wedge \psi^{n-1}$. Let $T_{\varepsilon}(Z[t])$ and $T_{\varepsilon}(R[t])$ be $\varepsilon$-tubes around $Z[t], R[t]$, respectively. Since $d d^{c} \log \xi$ is $C^{\infty}$ on the region $B_{\varepsilon}^{0}[t]=B[t]-T_{\varepsilon}(Z[t])-T_{\varepsilon}(R[t])$, Stokes' theorem gives

$$
\int_{B_{\varepsilon}^{0}[t]} d d^{c} \log \xi \wedge \psi^{n-1}=\int_{S[t]} d^{c} \log \xi \wedge \psi^{n-1}-\int_{\partial T_{\varepsilon}(Z[t])} d^{c} \log \xi \wedge \psi^{n-1}
$$

$$
-\int_{\partial T_{\varepsilon}(R[t])} d^{c} \log \xi \wedge \psi^{n-1} \text {. }
$$

Recall the residue formula for an analytic function $f$ :

$$
\lim _{\varepsilon \rightarrow 0} \int_{\partial T_{\varepsilon}(f)} d^{c} \log |f|^{2} \wedge \psi^{n-1}=\int_{(f)} \psi^{n-1}
$$

where $T_{\varepsilon}(f)$ is an $\varepsilon$-tube around the divisor $(f)$. Moreover, if $g$ is a function which does not vanish on $(f)$, we easily find that

$$
\lim _{\varepsilon \rightarrow 0} \int_{\partial T_{\varepsilon}(f)} d^{c} \log g \wedge \psi^{n-1}=0 .
$$


Now

$$
d^{c} \log \xi=(1 / m) d^{c} \log \left(a_{\alpha} \circ f\right)+(1 / m) d^{c} \log \left|s_{\alpha} \circ f\right|^{2}+d^{c} \log |J(f)|^{2} .
$$

Using this, together with the residue formula, we find that

(3) $\lim _{\varepsilon \rightarrow 0} \int_{B_{\varepsilon}^{0}[t]} d d^{c} \log \xi \wedge \psi^{n-1}=\int_{S[t]} d^{c} \log \xi \wedge \psi^{n-1}-\frac{1}{m} \int_{Z[t]} \psi^{n-1}-\int_{R[t]} \psi^{n-1}$.

On the other hand, since $d d^{c} \log |h|^{2}=0$ if $h$ is a nonvanishing holomorphic function, we find that

$$
\begin{aligned}
\int_{B_{\varepsilon}^{0}[t]} d d^{c} \log \xi \wedge \psi^{n-1} & =\frac{1}{m} \int_{B_{\varepsilon}^{0}[t]} d d^{c} \log \left(a_{\alpha} \circ f\right) \wedge \psi^{n-1} \\
& =\frac{1}{m} \int_{B_{\varepsilon}^{0}[t]} f^{*} c_{1}(L) \wedge \psi^{n-1} .
\end{aligned}
$$

Taking the limit in (4) and combining the result with (3), we obtain

(5) $\frac{1}{m} \int_{B[t]} f^{*} c_{1}(L) \wedge \psi^{n-1}+\frac{1}{m} \int_{Z[t]} \psi^{n-1}+\int_{R[t]} \psi^{n-1}=\int_{S[t]} d^{c} \log \xi \wedge \psi^{n-1}$.

For convenience, we introduce $K(t)=\int_{B[t]} f^{*} c_{1}(L) \wedge \psi^{n-1}$. Since $\psi$ has a singularity at the origin, we should show that $K(t)$ converges. This will be answered by the following lemma of Lelong (see [9, pp. 72-73]).

LemMa. Let $\theta$ be a closed positive current of type $(1,1)$. Then

$$
\int_{B\left[r_{1}\right]-B\left[r_{2}\right]} \theta \wedge \psi^{n-1}=\frac{1}{r_{1}^{2 n-2}} \int_{B\left[r_{1}\right]} \theta \wedge \beta^{n-1}-\frac{1}{r_{2}^{2 n-2}} \int_{B\left[r_{2}\right]} \theta \wedge \beta^{n-1} .
$$

(ii) If $\theta$ is $C^{\infty}$ at the origin, then the integral $\int_{B[r j} \theta \wedge \psi^{n-1}$ converges and $\int_{B[r]} \theta \wedge \psi^{n-1}=\left(r^{2 n-2}\right)^{-1} \int_{B[r]} \theta \wedge \beta^{n-1}$.

(iii) If $\theta$ is $C^{\infty}$ at the origin, then $\int_{B[r]} \theta \wedge \psi^{n-1} \leqq$ const $\cdot r^{2}$ for $r \leqq r_{0}$.

Since our notation is somewhat different from Lelong's we recall the proof. Since $B[r]$ is contractible, there is a current $\alpha$ such that $d \alpha=\theta$. An easy computation shows that $\psi^{n-1} \mid S[r]=\left(r^{2 n-2}\right)^{-1} \beta^{n-1}$. Then Stokes' theorem gives

$$
\begin{aligned}
\int_{B\left[r_{1}\right]-B\left[r_{2}\right]} \theta \wedge \psi^{n-1} & =\int_{S\left[r_{1}\right]} \alpha \wedge \psi^{n-1}-\int_{S\left[r_{2}\right]} \alpha \wedge \psi^{n-1} \\
& =\frac{1}{r_{1}^{2 n-2}} \int_{S\left[r_{1}\right]} \alpha \wedge \beta^{n-1}-\frac{1}{r_{2}^{2 n-2}} \int_{S\left[r_{2}\right]} \alpha \wedge \beta^{n-1}
\end{aligned}
$$

Another application of Stokes' theorem gives (i). If $\theta$ is $C^{\infty}$ at the origin, then for $r \leqq r_{0}$ sufficiently small, we have

$$
\begin{aligned}
\frac{1}{r^{2 n-2}} \int_{S[r]} \alpha \wedge \beta^{n-1} & =\frac{1}{r^{2 n-2}} \int_{B[r]} \theta \wedge \beta^{n-1} \\
& \leqq \frac{\text { const }}{r^{2 n-2}} \int_{B[r]} \beta^{n} \leqq \frac{\text { const }}{r^{2 n-2}} \cdot \operatorname{vol}(B[r]) \leqq \text { const } \cdot r^{2}
\end{aligned}
$$


Using (*) in (i) to compute the limit as $r_{2} \rightarrow 0$, we get (ii). The estimate (*) applied to (ii) gives (iii). Q.E.D.

Returning to the proof, we write (5) as

$$
\frac{1}{m} K(t)+\frac{1}{m} n(t)+n_{1}(t)=\int_{S[t]} d^{c} \log \xi \wedge \psi^{n-1} .
$$

We now integrate this from $\varepsilon$ to $r$ with respect to $d t / t$ to get

$$
\frac{1}{m} \int_{\varepsilon}^{r} K(t) \frac{d t}{t}+\frac{1}{m} \int_{\varepsilon}^{r} n(t) \frac{d t}{t}+\int_{\varepsilon}^{r} n_{1}(t) \frac{d t}{t}=\int_{\varepsilon}^{r} \frac{d t}{t} \int_{S[t]} d^{c} \log \xi \wedge \psi^{n-1} .
$$

Since $d t / t=d \log \|z\|$, Fubini's theorem gives

$$
\int_{\varepsilon}^{r} \frac{d t}{t} \int_{S[t]} d^{c} \log \xi \wedge \psi^{n-1}=\int_{B[t]-B[\varepsilon]} d \log \|z\| \wedge d^{c} \log \xi \wedge \psi^{n-1} .
$$

If $\alpha$ and $\beta$ are 1 -forms and $\phi$ is an $(n-1, n-1)$ form, then $d \alpha \wedge d^{c} \beta \wedge \phi$ $=-d^{c} \alpha \wedge d \beta \wedge \phi$. Therefore

$$
\begin{aligned}
d \log \|z\| \wedge d^{c} \log \xi \wedge \psi^{n-1} & =d \log \xi \wedge d^{c} \log \|z\| \wedge \psi^{n-1} \\
& =d\left(\log \xi \wedge d^{c} \log \|z\| \wedge \psi^{n-1}\right) .
\end{aligned}
$$

In the last step we have used the fact that $\psi^{n}=0$. Hence another application of Stokes' theorem to (7) reduces (6) to

$$
\frac{1}{m} \int_{\varepsilon}^{r} K(t) \frac{d t}{t}+\frac{1}{m} \int_{\varepsilon}^{r} n(t) \frac{d t}{t}+\int_{\varepsilon}^{r} n_{1}(t) \frac{d t}{t}=\int_{S[r]} \log \xi \sigma-\int_{S[\varepsilon]} \log \xi \sigma .
$$

Now $K(t)=\int_{B[t]} f^{*} c_{1}(L) \wedge \psi^{n-1} \leqq$ const $\cdot t^{2}$ by (iii) in the lemma above, so that the improper integral $\int_{0}^{r} K(t)(d t / t)$ converges. Moreover, $\int_{S[\varepsilon]} \log \xi \sigma$ is just the mean value of $\log \xi$ on $S[\varepsilon]$. Because of our normalization, $\log \xi(0)=0$, so that $\lim _{\varepsilon \rightarrow 0} \int_{S[\varepsilon]} \log \xi \sigma=0$.

Taking the limit as $\varepsilon \rightarrow 0$, the expression above becomes

$$
\frac{1}{m} \int_{0}^{r} K(t) \frac{d t}{t}+\frac{1}{m} N(t)+N_{1}(t)=\int_{S[r]} \log \xi \sigma .
$$

Proof of Proposition 1. The idea is to reinterpret $\int_{S[r]} \log \xi \sigma$ using concavity of the logarithm and polar coordinates. We give the proof for $T^{\#}$, the proof for $T$ being essentially the same. First, $\log \xi=n \log \xi^{1 / n}$. Then concavity of the logarithm gives

$$
\int_{S[r]} \log \xi \sigma \leqq n \log \int_{S[r]} \xi^{1 / n} \sigma .
$$

The formula for polar coordinates tells us that

$$
\left(\frac{2 \pi^{n}}{(n-1) !}\right) \int_{0}^{r} t^{2 n-1} d t \int_{S[t]} \xi^{1 / n} \sigma=\int_{B[r]} \xi^{1 / n} \Phi=\left(\frac{(2 \pi)^{m}}{n !}\right) \int_{B[r]} \xi^{1 / n} \beta^{n},
$$


where $2 \pi^{n} /(n-1)$ ! is the volume of the unit sphere in $C^{n}$ and $\Phi=\left((-1)^{1 / 2} / 2\right)^{n} d z_{1}$ $\wedge d \bar{z}_{1} \wedge \cdots \wedge d z_{n} \wedge d \bar{z}_{n}$ is the standard euclidean volume form on $\boldsymbol{C}^{n} \cong \boldsymbol{R}^{2 n}$. Differentiating the above identity, we get

$$
\left(\frac{2^{n-1}}{n}\right) \frac{1}{r^{2 n-1}} \frac{d}{d r} \int_{B[r]} \xi^{1 / n} \beta^{n}=\int_{S[r]} \xi^{1 / n} \sigma .
$$

Applying these remarks to (8), we get

$$
\frac{1}{m} \int_{0}^{r} K(t) \frac{d t}{t}+\frac{1}{m} N(t)+N_{1}(t) \leqq n \log \left[\left(\frac{2^{n-1}}{n}\right) \frac{1}{r^{2 n-1}} \frac{d T^{\#}}{d r}\right] .
$$

Now the left-hand side of (9) is nonnegative, which implies that

$$
\left(\frac{2^{n-1}}{n}\right) \frac{1}{r^{2 n-1}} \frac{d T^{\#}}{d r} \geqq 1 \text {. }
$$

Integrating the inequality $d T^{\#} / d r \geqq\left(n / 2^{n-1}\right) r^{2 n-1}$ from 0 to $r$ gives $T^{\#}(r)$ $\geqq\left(n /(2 n-1) 2^{n-1}\right) r^{2 n}$, which we write as $T^{\#}(r) \geqq l_{0} r^{2 n}$.

Proof of Proposition 2. The idea is to reinterpret the term $\int_{0}^{r} K(t) d t / t$ in (9) using the order function $T^{\#}$. It is here that the assumption that $L$ be positive is critical. We first remark that if $G$ is a positive semidefinite hermitian matrix, then $\operatorname{det} G^{1 / n} \leqq$ trace $G / n$. This follows from the theorem on the geometric and arithmetic means, $\left(\lambda_{1}, \ldots, \lambda_{n}\right)^{1 / n}=\left(\lambda_{1}+\cdots+\lambda_{n}\right) / n$ applied to the eigenvalues of $G$. Now recall that if $\theta$ is a positive $C^{\infty}(1,1)$ form, we have the relation between the projective and euclidean trace (see $[9, \mathrm{pp} .72-73]$ )

$$
\int_{B[r]} \theta \wedge \psi^{n-1}=\frac{1}{r^{2 n-2}} \int_{B[r]} \theta \wedge \beta^{n-1}
$$

Now let $\theta=f^{*} c_{1}(L)=\left((-1)^{1 / 2} / 4 \pi\right) \sum g_{k l} d z_{k} \wedge d \bar{z}_{l}$, where the hermitian matrix $G=\left(g_{k l}\right)$ is positive definite. An easy computation gives $\theta \wedge \beta^{n-1}=($ trace $G) \beta^{n}$. Substituting into (10), we get

$$
\begin{aligned}
\int_{B[t]} f^{*} c_{1}(L) \wedge \psi^{n-1} & =\frac{1}{t^{2 n-2}} \int_{B[t]}(\operatorname{trace} G) \beta^{n} \\
& \geqq \frac{n}{t^{2 n-2}} \int_{B[t]}(\operatorname{det} G)^{1 / n} \beta^{n}
\end{aligned}
$$

Now $V$ is compact and $c_{1}(L)^{n}$ is an everywhere positive $(n, n)$ form, so there is a constant $k>0$ such that $c_{1}(L)^{n} \geqq\left(n ! k /(4 \pi)^{n}\right) \Omega$. Pulling this back to $B[t]$, we get $f^{*} c_{1}(L)^{n} \geqq\left(n ! k /(4 \pi)^{n}\right) f^{*} \Omega$, or $(\operatorname{det} G) \beta^{n} \geqq k \xi \beta^{n}$. Therefore det $G \geqq k \xi$ and

$$
(\operatorname{det} G)^{1 / n} \geqq k^{1 / n} \xi^{1 / n} \text {. }
$$

Substituting this into (11) gives

$$
\int_{B[t]} f^{*} c_{1}(L) \wedge \psi^{n-1} \geqq \frac{n k^{1 / n}}{t^{2 n-2}} \int_{B[t]} \xi^{1 / n} \beta^{n}
$$


Recalling the definitions of $K$ and $T^{\#}$, the estimate (9) becomes

$$
\left(\frac{n k^{1 / n}}{m}\right) \int_{0}^{r} T^{\#}(t) \frac{d t}{t^{2 n-1}} \frac{1}{m} N(r)+N_{1}(r) \leqq n \log \left[\frac{1}{r^{2 n-1}} \frac{d T^{\#}}{d r}\right]+l_{1}
$$

where $l_{1}=\log \left(2^{n-1} / n\right) \geqq 0$. Note that for $t \leqq t_{0}$,

$$
\int_{B[t]} \xi^{1 / n} \beta^{n} \leqq \text { const } \cdot \operatorname{vol}(B[t]) \leqq \text { const } \cdot t^{2 n}
$$

so that the integral $\int_{0}^{r} T^{\#}(t) d t / t^{2 n-1}$ converges. This ends the proof of Proposition 2.

Proof of the Schottky-Landau theorem. Before giving the formal proof, we will give a heuristic argument. First, the basic estimate gives

$$
\frac{k^{1 / n}}{m} \int_{0}^{r} T^{\#}(t) \frac{d t}{t^{2 n-1}} \leqq n \log \left[\frac{1}{r^{2 n-1}} \frac{d T^{\#}}{d r}\right]+l_{1} .
$$

Suppose that $T^{\#}$ is extremely nice, let us say $T^{\#}(r)=r^{\alpha+2 n}$. By Proposition 1, we know $\alpha \geqq 0$. Substituting into (8) we obtain

$$
\frac{k^{1 / n}}{m} \int_{0}^{r} t^{\alpha+1} d t \leqq \log \left[(\alpha+2 n) t^{\alpha}\right]+l_{1},
$$

hence

$$
\text { const } \cdot t^{\alpha+2} \leqq \text { const } \cdot \log t+\text { const. }
$$

The left-hand side of this equation grows much faster than the right-hand side, so it is valid only for $r \leqq R_{\max }$ for some $R_{\max }<\infty$. A crude version of the SchottkyLandau theorem can be obtained by a slight refinement of this argument.

Proposition. If $V$ is of general type and $f: B(R) \rightarrow W$ is nondegenerate, then $R<\infty$.

Proof. We recall a standard calculus lemma of value distribution theory (see $[11$, p. 54]).

Lemma. Let $\psi$ be a continuously differentiable, positive, increasing function on $\left[r_{0}, \infty\right]$, where $r_{0} \geqq 1$. Then $\psi^{\prime} \leqq \psi^{\beta}$ for any $\beta>1$ on $J=\left[r_{0}, \infty\right]-I$, where $I$ is an open set such that $\int_{I} d \log x<\infty$.

One easily checks that $T^{\#}(r)$ satisfies the hypotheses of the lemma $\left({ }^{11}\right)$. The inequality (13) yields

$$
A \int_{0}^{r} T^{\#}(t) \frac{d t}{t^{2 n-1}} \leqq \log \left[\frac{T^{\# 2}(r)}{r^{2 n-1}}\right]+l_{1} \quad \text { on } J_{1},
$$

${ }^{(11)}$ For example, $d T^{\#} / d r=$ const $\cdot r^{2 n-1} \int_{S[r]} \xi^{1 / n} \sigma$. Since $\xi^{1 / n}$ is continuous, Stokes' theorem shows $\int_{S[r]} \xi^{1 / n} \sigma$ is a continuous function of $r$, so that $d T \# \mid d r$ is continuous. 
where $A=k^{1 / n} / m$. Introduce $S(r)=\int_{0}^{r} T^{\#}(r) d t / t^{2 n-1}$ and write the above equation as

$$
A S(r) \leqq \log T^{\#}(r)+\log \left(T^{\#}(r) / r^{2 n-1}\right)+l_{1} \text { on } J_{1} .
$$

Now $d S / d r=T^{\#}(r) / r^{2 n-1}$, and the lemma gives $d S / d r \leqq S^{2}$ on $J_{2}$. Therefore we get

$$
A S(r) \leqq \log T^{\#}(r)+2 \log S(r)+l_{1} \quad \text { on } J_{1} \cap J_{2} .
$$

We rewrite this as

$$
A S(r) \leqq(2 n-1) \log r+\log (d S / d r)+2 \log S(r)+l_{1}
$$

and apply $d S / d r \leqq S^{2}$ on $J_{2}$ once more to get

$$
A S(r) \leqq(2 n-1) \log r+4 \log S(r)+l_{1} \quad \text { on } J_{1} \cap J_{2} .
$$

Dividing by $S(r)$ we get

$$
A \leqq(2 n-1) \frac{\log r}{S(r)}+\frac{4 \log S(r)}{S(r)}+\frac{l_{1}}{S(r)} \quad \text { on } J_{1} \cap J_{2}
$$

Now $T^{\#}(r) \geqq l_{0} r^{2 n}$, so $S(r) \geqq\left(l_{0} / 2\right) r^{2}$. Moreover, the complement of any set of finite logarithmic measure contains a sequence $\left\{r_{j}\right\}$ such that $r_{j} \rightarrow \infty$. Therefore we find that

$$
0<A \leqq \frac{2(2 n-1)}{l_{0}} \frac{\log r_{j}}{r_{j}^{2}}+\frac{4 \log S\left(r_{j}\right)}{S\left(r_{j}\right)}+\frac{2 l_{1}}{l_{0} r_{j}^{2}}
$$

Using l'Hôpital's rule to compute $\lim \left(\log S\left(r_{j}\right) / S\left(r_{j}\right)\right)$, we see that the right-hand side of (15) tends to zero as $r_{j} \rightarrow \infty$, thus the inequality is valid only for $r \leqq R_{\max }$ $<\infty$. Q.E.D.

Proof of the precise Schottky-Landau theorem. The problem with the above "proof" is that the choice of $J_{1}$ and $J_{2}$ is not a priori independent of the order function $T^{\#}$, which certainly depends on $f$. The precise Schottky-Landau theorem is proved by a more precise analysis of the integrated order function $S(r)$ $=\int_{0}^{r} T^{\#}(t) d t / t^{2 n-1}$ (see [8]). For convenience, we write $\Omega(r)=\left(r^{2 n-1}\right)^{-1} d T^{\#} / d r$. Then (13) becomes

$$
A S(r) \leqq n \log B \Omega(r)
$$

where $B=e^{l_{1}}=2^{n-1} / n$.

The first step in the proof is to obtain a tentative bound for $R_{\max }$ by assuming

$$
\Omega(r) \leqq r^{2 n-1} S(r)^{4} .
$$

Assuming (*), inequality (16) becomes

$$
S(r) \leqq(n / A)(\log B+(2 n-1) \log r+4 \log S(r)) .
$$


Dividing by $S(r)$ and simplifying constants we get

$$
1 \leqq \frac{n}{A}\left(\frac{l_{1}}{S(r)}+(2 n-1) \frac{\log r}{S(r)}+\frac{4 \log S(r)}{S(r)}\right) .
$$

Using $S(r) \geqq\left(l_{0} / 2\right) r^{2}$ and $\log x \leqq e^{-1} x$ for $x>0$, the first two terms are dominated by $\left(2 l_{1} / l_{0}\right) r^{-2},\left(2(2 n-1) e^{-1} / l_{0}\right) r^{-1}$, respectively. Writing $(4 \log S) / S=\left(8 \log S^{1 / 2}\right) / S$ $\leqq 8 e^{-1} / S^{1 / 2} \leqq 8(2)^{1 / 2} e^{-1} / l_{0}^{1 / 2} r$, and assuming $r \geqq 1$, the above estimate becomes

$$
1 \leqq \frac{n}{A}\left\{\frac{2 l_{1}}{l_{0}}+\frac{2(2 n-1) e^{-1}}{l_{0}}+\frac{8(2)^{1 / 2} e^{-1}}{l_{0}^{1 / 2}}\right\} \frac{1}{r}
$$

We write this as $1 \leqq r_{0}\left(k, n, p_{0}\right) r^{-1}$ where $r_{0}$ is a constant depending only on $n, k$, and the point $p_{0}$. Note that we have chosen $r_{0} \geqq 1$. We conclude that if $\Omega(r)$ $\leqq r^{2 n-1} S(r)^{4}$, then $r \leqq r_{0}$.

Now assume that $r>r_{0}$. By what we have just shown, we know that one of the following must hold:

(a) $T^{\# 2}(r)<r^{2 n-1} \Omega(r)$,

(b) $r^{2 n-1} S^{2}(r)<T^{\#}(r)$.

Suppose that (a) holds on an interval $I$. Using $\Omega(r)=\left(r^{2 n-1}\right)^{-1} d T^{\#} / d r$ we find that $d r<d T^{\# /} / T^{\# 2}$. Suppose (b) holds on an interval $J$. Using $d S / d r=T^{\#}(r) / r^{2 n-1}$ we find $d r<d S / S^{2}$. Since both $T$ and $S$ are nondecreasing, this implies that on the interval $\left(r_{0}, R_{\max }\right)$ we have $d r<d T^{\# /} / T^{\# 2}+d S / S^{2}$. Integrating this inequality we obtain

$$
\begin{aligned}
& r-r_{0}=\int_{r_{0}}^{r} d t<\int_{r_{0}}^{r} d\left(-\frac{1}{T^{\#}}\right)+\int_{r_{0}}^{r} d\left(-\frac{1}{S}\right), \\
& r-r_{0}<\frac{1}{T^{\#}\left(r_{0}\right)}+\frac{1}{S\left(r_{0}\right)} \leqq \frac{l_{0}^{-1}}{r_{0}^{2 n}}+\frac{2 l_{0}^{-1}}{r_{0}^{2}} .
\end{aligned}
$$

Since $r_{0} \geqq 1$, this yields $R_{\max } \leqq r_{0}+3 / l_{0}$. Thus we have obtained a bound on $R_{\max }$ depending only on $n, k$, and $p_{0}$.

III. A degeneracy theorem for holomorphic maps $f: \boldsymbol{C}^{n} \rightarrow \boldsymbol{P}_{n}-D$. In this section we discuss an $n$-dimensional generalization of Picard's theorem. The first result is the following:

THEOREM 1. Let $D \subseteq \boldsymbol{P}_{n}$ be a nonsingular divisor of degree $d \geqq n+3$. Then any holomorphic mapping $f: \boldsymbol{C}^{n} \rightarrow \boldsymbol{P}_{n}-D$ is totally degenerate, i.e. $|J(f)| \equiv 0$.

In dimension one this says that any entire function $f: \boldsymbol{C} \rightarrow \boldsymbol{P}_{1}-\{$ four points $\}$ is constant, a crude version of Picard's theorem. Another way of stating Theorem 1 is that any nondegenerate holomorphic map $f: \boldsymbol{C}^{n} \rightarrow \boldsymbol{P}_{n}$ must intersect $D$ if $D$ is smooth and has high enough degree.

Proof of the theorem. We construct a cyclic branched cover $W_{n}$ of $\boldsymbol{P}_{n}$ satisfying

(a) $W_{n} \stackrel{\pi}{\rightarrow} \boldsymbol{P}_{n}$ is branched exactly along $D$.

(b) $W_{n}$ is a nonsingular hypersurface of degree $d$ in $\boldsymbol{P}_{n+1}$. 
If $f_{d}\left(z_{0}, \ldots, z_{n}\right)=0$ is a homogeneous equation for $D$, one easily verifies that such a $W_{n}$ is given by $F\left(z_{0}, \ldots, z_{n+1}\right)=z_{n+1}^{d}-f_{d}\left(z_{0}, \ldots, z_{n}\right)$. Note that $W_{n}-D=W_{n}$ $-\pi^{-1}(D)$ because the cover is cyclic. Because of condition (a), $\pi: W_{n}-D \rightarrow \boldsymbol{P}_{n}-D$ is a covering $\operatorname{map}\left({ }^{12}\right)$, so $f$ lifts to a $\operatorname{map} f^{\prime}: C^{n} \rightarrow W_{n}-D \subseteq W_{n}$.

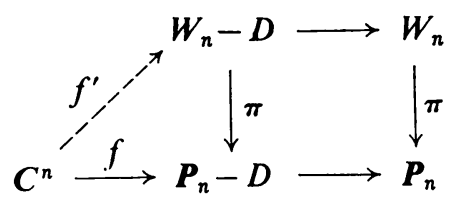

The idea is now to apply Griffiths' theorem on canonical algebraic varieties (see [3]), which implies that any holomorphic map $f: C^{n} \rightarrow W_{n}$ is totally degenerate if $K_{W_{n}}$ is very ample. Thus it remains to show that the cyclic cover constructed above has very ample canonical bundle.

To see this recall that a line bundle $L$ on a variety $V$ is very ample if its global sections give an immersion of $V$ into $\boldsymbol{P}_{N}$. The immersion $g_{L}$ is constructed by taking a basis $s^{0}, \ldots, s^{N}$ for the global sections of $L$ and sending $x \rightarrow\left[s^{0}, \ldots, s^{N}\right]$, where $\left[w_{0}, \ldots, w_{N}\right]$ are homogeneous coordinates on $\boldsymbol{P}_{N}$. Moreover, if $W \subset V$ is a submanifold of $V$, then the global sections of $L$ on $V$ restrict to global sections of $L$ on $W$, so that if $L$ is very ample on $V$, it is very ample on any subvariety $W$. By explicit computation, we see that any positive multiple of the hyperplane bundle $[H]$ on $\boldsymbol{P}_{n+1}$ is very ample, and hence very ample when restricted to any subvariety of $\boldsymbol{P}_{n+1}$. Now we can explicitly compute $K_{W}$ from the adjunction formula $K_{W}=\left.K_{P_{n+1}} \otimes[W]\right|_{W}$, where $[W]$ is the line bundle determined by the divisor $W$. Recall that $K_{P_{n+1}}=[H]^{-(n+2)}$. Moreover, $W$ is linearly equivalent to $[H]^{d}$. To see this, just observe that $g=f_{d} / z_{0}^{d}$ exhibits the linear equivalence. We conclude that $K_{W}=\left.[H]^{d-(n+2)}\right|_{W}$, so that $K_{W}$ is very ample if $d>n+2$. Q.E.D.

To state the next theorem, we let $D \subseteq V_{n}$ be a possibly singular divisor. $D$ is said to have good singularities if for each point $x \in D$ there are local analytic coordinates $z_{1}, \ldots, z_{n}$ on $V_{n}$ centered at $x$ such that a local equation for $D$ is $z_{1} \ldots z_{k}=0$. Thus the singularities of $D$ are locally normal crossings. For example, a union of nonsingular hypersurfaces meeting transversely with no more than $n$ components passing through any point is such a divisor. An interesting special case is the union of hyperplanes in general position. Other examples are surfaces in $\boldsymbol{P}_{\mathbf{2}}$ with a nonsingular double curve, etc.

THEOREM $1^{\prime}$. Let $D \subseteq \boldsymbol{P}_{n}$ be a divisor of degree $d \geqq n+3$ having good singularities. Let $f: B(R) \rightarrow \boldsymbol{P}_{n}-D$ be a nondegenerate holomorphic map normalized so that $f(0)=p_{0} \in \boldsymbol{P}_{n}-D$ and $|J(f)(0)|=1$. Then there is an absolute constant $R_{\max }\left(n, p_{0}\right)$ such that $R \leqq R_{\max }\left(n, p_{0}\right)$.

${ }^{(2)}$ In fact, $\pi_{1}\left(\boldsymbol{P}_{n}-D\right) \cong \boldsymbol{Z}_{d}$. The covering transformations are given by $\left[z_{0}, \ldots, z_{n+1}\right]$ $\rightarrow\left[z_{0}, \ldots, p z_{n+1}\right]$, where $p=\exp (2 \pi i / d)$. Hence $W_{n}-D$ is the universal cover of $\boldsymbol{P}_{n}-D$. 
As an immediate consequence we have

CoRollary 1. Let $D \subseteq \boldsymbol{P}_{n}$ be a divisor of degree $d \geqq n+3$ with good singularities. Then any holomorphic map $f: \boldsymbol{C}^{n} \rightarrow \boldsymbol{P}_{n}-D$ is totally degenerate.

The proof is somewhat more complicated than in the previous case, although the idea is the same. We begin by defining a cyclic branched cover $W_{n} \stackrel{\pi}{\rightarrow} \boldsymbol{P}_{n}$ which is branched exactly along $D$. Just as before, $W_{n}$ is defined by $z_{n+1}^{d}-f_{d}\left(z_{0}, \ldots, z_{n}\right)=0$. The technical problem is that $W_{n}$ has singularities lying over the singularities of $D$. By means of explicit quadratic transformations we desingularize $W_{n}$ to obtain an algebraic manifold $W_{n}^{\prime} \stackrel{\pi^{\prime}}{\rightarrow} \boldsymbol{P}_{n}$. Because the singularities of $W_{n}$ lie in the singular locus of $D, W_{n}^{\prime}-\pi^{\prime-1}(D) \rightarrow W_{n}-\pi^{-1}(D)$. Moreover, $\pi^{\prime}: W_{n}^{\prime}-\pi^{\prime-1}(D) \rightarrow \boldsymbol{P}_{n}-D$ is a covering map, so we get a lifting $f^{\prime}: C^{n} \rightarrow W_{n}^{\prime}-\pi^{\prime-1}(D) \subseteq W_{n}^{\prime}$. If we can show that $W_{n}^{\prime}$ is of general type, Kodaira's theorem will apply to give Theorem 1'.

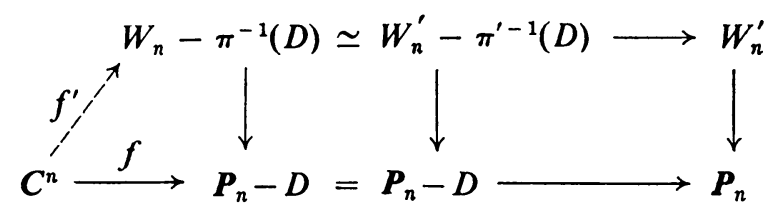

The theorem is therefore proved modulo the lemma:

Lemma 1. Let $D \subseteq \boldsymbol{P}_{n}$ be a divisor with good singularities such that $\operatorname{deg}(D) \geqq n+3$. Let $W_{n}^{\prime}$ be a desingularization of the standard d-fold cyclic cover $W_{n}$ of $\boldsymbol{P}_{n}$ branched exactly along $D$. Then $W_{n}^{\prime}$ is of general type.

To give the proof of the lemma, we review the notion of rational differential forms and the residue operator [4]. Next we discuss the resolution of singularities. Then we will be able to calculate the so-called adjoint conditions which the singularities of $W_{n}$ impose upon the rational differential forms in the ambient projective space, from which the lemma will follow.

(A) The residue operator. Let $V_{n+1}$ be an $(n+1)$-dimensional complex manifold, and $W \subseteq V_{n+1}$ a nonsingular divisor. Given a rational $(n+1)$-form $w$ with a first order pole along $W$, we want to define a holomorphic $n$-form $R(\omega)$ on $W$. We first solve the problem locally. The global result will follow by taking sections of the appropriate sheaves. Thus we set $V_{n+1}=P_{n+1}$, the $(n+1)$-dimensional polycylinder. On $P_{n+1}, \omega=\left(h d z_{1} \wedge \cdots \wedge d z_{n+1}\right) / f$, where $f$ is a local defining equation for $W$. Since $W$ is nonsingular, we may assume by shrinking $P_{n+1}$ that one of the partial derivatives, say $\partial f / \partial z_{n+1}$, is nowhere zero on $W$. Then we have

hence

$$
\left(\frac{\partial f}{\partial z_{n+1}}\right)^{-1} d f=\left(\frac{\partial f}{\partial z_{n+1}}\right)^{-1}\left(\frac{\partial f}{\partial z_{1}} d z_{1}+\cdots+\frac{\partial f}{\partial z_{n}} d z_{n}\right)+d z_{n+1}
$$

$$
\omega=\frac{h d z_{1} \wedge \cdots \wedge d z_{n}}{\partial f / \partial z_{n+1}} \wedge \frac{d f}{f} .
$$


Define

$$
R(\omega)=\frac{h d z_{1} \wedge \cdots \wedge d z_{n}}{\partial f / \partial z_{n+1}}
$$

i.e. we integrate out the logarithmic term $d f / f$. One easily checks (see [4]) that the definition is independent of the choice of local defining equation, etc. The result of this is the Poincaré residue sequence

$$
0 \longrightarrow K_{V} \longrightarrow K_{V} \otimes[W] \stackrel{R}{\longrightarrow} K_{W} \longrightarrow 0 .
$$

The exact cohomology sequence globalizes this to give

$$
H^{0}\left(K_{V}\right) \longrightarrow H^{0}\left(K_{V} \otimes[W]\right) \stackrel{R}{\longrightarrow} H^{0}\left(K_{W}\right)
$$

(B) Rational differential forms. A rational differential form on $\boldsymbol{P}_{n+1}$ with a first order pole along $D$ is just a section of $K_{\boldsymbol{P}_{n+1}} \otimes[D]=K_{\boldsymbol{P}_{n+1}}(D)$. If $\boldsymbol{C}^{n+1} \subseteq \boldsymbol{P}_{n+1}$ is an affine piece of $\boldsymbol{P}_{n+1}$ given by $z_{0} \neq 0$, then a section $\omega \in \Gamma\left(K_{\boldsymbol{P}_{n+1}}(D)\right)$ is given by

$$
\omega=\left(P d z_{1} \wedge \cdots \wedge d z_{n+1}\right) / Q
$$

where $Q$ is the affine equation for $D$ and $P$ is a polynomial of degree $d-(n+2)$.

(C) Resolution of singularities. Suppose that $Z \subseteq V$ is a nonsingular subvariety of codimension $\geqq 2$. Then there are a variety $V^{\prime}$ and a holomorphic map $p: V^{\prime} \rightarrow V$ such that

(a) $p: V^{\prime}-p^{-1}(Z) \rightarrow V-Z$ is biholomorphic,

(b) $p^{-1}(Z)=\boldsymbol{P}(N(Z))$.

Here $N(Z)$ is the normal bundle of $Z$ and $\boldsymbol{P}(N(Z))$ is the variety in $V$ obtained by replacing each fiber $N_{z}$ by the associated projective space $\boldsymbol{P}\left(N_{z}\right)$. Note that $V^{\prime}$ is nonsingular and $\boldsymbol{P}(N(Z))$ is a divisor. If $W \subseteq V$ is a singular subvariety of $V$, we set $W^{\prime}=$ closure of $p^{-1}(W-Z)$. Notice that $p^{-1}(W)=W^{\prime} \cup \mathscr{E}$, where $\mathscr{E}=\boldsymbol{P}(N(Z))$ is the so-called exceptional set. We call $p^{-1}(W)$ the total transform of $W$ and $W^{\prime}$ the proper transform. We say that $V^{\prime}, W^{\prime}$ are obtained by blowing up $V, W$, respectively, along $Z$. The aim is to resolve the singularities of $V$ by a sequence of such transformations.

In order to perform the necessary computations, we need an explicit local description of the map $p$. Therefore, let $P_{n}$ be a polycylindrical neighborhood of a point $x \in V$ such that $P_{n} \cap Z$ is defined by $z_{1}=0, \ldots, z_{k}=0$ where $k=\operatorname{codim} Z$. Then $p^{-1}\left(P_{n}\right)=P_{n}^{\prime}$ is defined as a subvariety of $P_{n} \times \boldsymbol{P}_{k-1}$ as follows. Let $w_{1}, \ldots, w_{k}$ be homogeneous coordinates on $\boldsymbol{P}_{k-1}$. Then $P_{n}^{\prime}=\left\{\left(z_{1}, \ldots, z_{n}, w_{1}, \ldots, w_{k}\right) \mid w_{i} z_{j}\right.$ $\left.=w_{j} z_{i}, 1 \leqq i, j \leqq k\right\}$. Now $P_{n} \times \boldsymbol{P}_{k-1}$ is covered by open sets $P_{n} \times U_{\alpha}$, where $U_{\alpha}$ $=P_{k-1}-\left\{w_{\alpha}=0\right\}$. Coordinate functions on $U_{\alpha}$ are $\xi_{j}^{\alpha}=w_{j} / w_{\alpha}, 1 \leqq j \leqq k, j \neq \alpha$. Thus coordinates on $P_{\alpha}$ are given by $\left\{z_{\alpha}, \xi_{1}^{\alpha}, \ldots, \hat{\xi}_{\alpha}^{\alpha}, \ldots, \xi_{k}^{\alpha}, z_{k+1}, \ldots, z_{n}\right\}\left({ }^{13}\right)$. The map

$\left({ }^{13}\right)$ Here $^{\wedge}$ denotes omission. 
$p: P_{n}^{\prime} \rightarrow P_{n}$ is induced by the projection $P_{n} \times \boldsymbol{P}_{k-1} \rightarrow P_{n}$. Therefore, $p$ is given locally by

$$
p_{\alpha}\left(z_{\alpha}, \xi_{1}^{\alpha}, \ldots, \xi_{k}^{\alpha}, z_{k+1}, \ldots, z_{n}\right)=\left(z_{\alpha} \xi_{1}^{\alpha}, \ldots, z_{\alpha}, \ldots, z_{\alpha} \xi_{k}^{\alpha}, z_{k+1}, \ldots, z_{n}\right) .
$$

Since this notation is somewhat disgusting and cumbersome, we usually write

$$
p_{\alpha}\left(\eta_{1}, \ldots, \eta_{n}\right)=\left(\eta_{\alpha} \eta_{1}, \ldots, \eta_{\alpha}, \ldots, \eta_{\alpha} \eta_{k}, \eta_{k+1}, \ldots, \eta_{k}\right) .
$$

EXAmple. Consider the variety defined by $f=z_{1}^{2}+z_{2}^{2}=0$ in $P_{3}$. We perform a quadratic transformation (blowing up) centered along $\left\{z_{1}=0\right\} \cap\left\{z_{2}=0\right\}$. One of the projection maps is given by $p\left(\eta_{1}, \eta_{2}, \eta_{3}\right)=\left(\eta_{1}, \eta_{1} \eta_{2}, \eta_{3}\right)$. The total transform is $f \circ p=\eta_{1}^{2}+\eta_{1}^{2} \eta_{2}^{2}=\eta_{1}^{2}\left(1+\eta_{2}^{2}\right)$. The smooth variety $1+\eta_{2}^{2}=0$ is the proper transform, and $\eta_{1}^{2}=0$ is the exceptional set.

(D) Calculation of the adjoint conditions. The problem now is to find a $W^{\prime} \subseteq \boldsymbol{P}_{n+1}^{\prime}$ which desingularizes $W$, and from this to calculate $K_{W^{\prime}}$. The residue operator gives sections of $K_{W^{\prime}}$ from sections of $K_{P_{n+1}^{\prime}}\left(W^{\prime}\right)$, so what we really need is a description of the global sections of $K_{P_{n+1}^{\prime}}\left(W^{\prime}\right)$. If $\omega$ is a global section of $K_{P_{n+1}}(W)$, then $p^{*} \omega$ has a pole along the total transform $W^{\prime} \cup \mathscr{E}$. Therefore we must impose restrictions on sections of $K_{P_{n+1}}(W)$ such that $p^{*} \omega$ has a simple pole on $W^{\prime}$ but is holomorphic elsewhere. These are the so-called adjoint conditions on $W$. If we look at the problem locally, what we really want is a description of the subsheaf $p_{*} K_{\boldsymbol{P}_{n+1}^{\prime}}\left(W^{\prime}\right) \subseteq K_{\boldsymbol{P}_{n+1}}(W)$. Then every global section of $p_{*} K_{\boldsymbol{P}_{n+1}^{\prime}}\left(W^{\prime}\right)$ pulls back to a global section of $K_{P_{n+1}}(W)$, and this will give us what we need. The calculation of the adjoint conditions is thus reduced to an essentially local problem. Our immediate goal is to prove

Lemma 2. For $W$ as in Theorem $1^{\prime}, p_{*} K_{\boldsymbol{P}_{n+1}^{\prime}}\left(W^{\prime}\right)=K_{\boldsymbol{P}_{n+1}}(W)$, i.e. no adjoint conditions are imposed.

The proof of the lemma is somewhat tedious, so we give an example to illustrate how the computations go.

EXAMPLE. Let $\boldsymbol{W \subseteq \boldsymbol { P } _ { n + 1 }}$ be a hypersurface of degree $d$ which is nonsingular except at the point $x$. Suppose that a local analytic equation for $W$ at $x$ is $f=z_{1}^{k}+$ $\cdots+z_{n+1}^{k}=0$. A rational $(n+1)$-form is given locally by

$$
\omega=\left(h d z_{1} \wedge \cdots \wedge d z_{n+1}\right) /\left(z_{1}^{k}+\cdots+z_{n+1}^{k}\right) .
$$

Consider the quadratic transformation centered at $x\left({ }^{14}\right) \cdot p: \boldsymbol{P}_{n+1}^{\prime} \rightarrow \boldsymbol{P}_{n+1}$ is given locally by $n+1$ projections

$$
\begin{gathered}
p_{1}\left(\eta_{1}, \ldots, \eta_{n+1}\right)=\left(\eta_{1}, \eta_{1} \eta_{2}, \ldots, \eta_{1} \eta_{n+1}\right), \\
\vdots \vdots \\
p_{n+1}\left(\eta_{1}, \ldots, \eta_{n+1}\right)=\left(\eta_{n+1} \eta_{1}, \ldots, \eta_{n+1} \eta_{n}, \eta_{n+1}\right) .
\end{gathered}
$$

$\left({ }^{14}\right)$ I.e., we blow up $x$ and replace it by a $\boldsymbol{P}_{n}$. 
The total transform of $W$ near $x$ is given in pieces, for example by $f \circ p_{1}$ $=\eta_{1}^{k}+\eta_{1}^{k} \eta_{2}^{k}+\cdots+\eta_{1}^{k} \eta_{n+1}^{k}=\eta_{1}^{k}\left(1+\eta_{2}^{k}+\eta_{n+1}^{k}\right)$. The proper transform is $f^{1}=1+\eta_{2}^{k}$ $+\cdots+\eta_{n+1}^{k}$, which is nonsingular. Moreover, we find that

$$
p_{1}^{*} \omega=\frac{h \circ p \eta_{1}^{n} d \eta_{1} \wedge \cdots \wedge d \eta_{n+1}}{f \circ p_{1}}=\frac{h \circ p \eta_{1}^{n-k} d \eta_{1} \wedge \cdots \wedge d \eta_{n+1}}{f^{1}} .
$$

Therefore, if $n \geqq k$, the piece of the resolution given by $p_{1}$ imposes no adjoint conditions on $\omega$. By symmetry, we see that the other pieces of the resolution give the same result. We conclude that $W$ can be resolved in one step by $p: \boldsymbol{P}_{n+1}^{\prime} \rightarrow \boldsymbol{P}_{n+1}$ and that no adjoint conditions are imposed, i.e. $p_{*} K_{\boldsymbol{P}_{n+1}}(W)=K_{\boldsymbol{P}_{n+1}}(W)$. Let $\omega$ be the globally defined rational $(n+1)$-form on $\boldsymbol{P}_{n+1}$ given by

$$
\omega=\left(P d z_{1} \wedge \cdots \wedge d z_{n+1}\right) / Q
$$

on a standard affine open $\boldsymbol{C}^{n+1} \subseteq \boldsymbol{P}_{n+1}$. Thus $Q$ is an affine equation for $W$ and $P$ is a polynomial of degree $d-(n+2)$. The $p^{*} \omega$ is a globally defined rational $(n+1)-$ form on $\boldsymbol{P}_{n+1}^{\prime}$ with $W^{\prime}$ as simple polar locus. Since $W^{\prime}$ is nonsingular, the residue operation applied to $p^{*} \omega$ gives a holomorphic $n$-form on $W^{\prime}$. Because of the explicit form of the residue operation given in (A), we see that the divisor of $R\left(p^{*} \omega\right)$ on $W^{\prime}-\mathscr{E}$ is just the divisor $P \circ p=0$.

Proof of Lemma 2. We first consider the case where $\underset{\sim}{\boldsymbol{\pi}} \rightarrow \boldsymbol{P}_{n}$ is branched over $D$ such that at most two branches of $D$ meet at any point. Thus a local analytic equation for $W$ is $f=z_{n+1}^{d}-z_{1} z_{2}$. We blow up along the locus $\left\{z_{1}=0\right\}$ $\cap\left\{z_{n+1}=0\right\}\left({ }^{15}\right)$. Since $p^{-1}\left(P_{n+1}\right) \subseteq P_{n+1} \times \boldsymbol{P}_{1}$, the projection is given in two pieces:

$$
\begin{aligned}
& p_{A}\left(\eta_{1}, \ldots, \eta_{n+1}\right)=\left(\eta_{n+1} \eta_{1}, \ldots, \eta_{n+1}\right), \\
& p_{B}\left(\eta_{1}, \ldots, \eta_{n+1}\right)=\left(\eta_{1}, \eta_{2}, \ldots, \eta_{1} \eta_{n+1}\right) .
\end{aligned}
$$

A rational $(n+1)$-form on $P_{n+1}$ with simple pole on $W$ is represented on $P_{n+1}$ by $\omega=\left(h d z_{1} \wedge \cdots \wedge d z_{n+1}\right) / f$. Now the total transform of $f$ by $p_{A}$ is $f \circ p_{A}=\eta_{n+1}^{d}$ $-\left(\eta_{n+1} \eta_{1}\right) \eta_{2}$ and the proper transform is $f^{A}=\eta_{n+1}^{d-1}-\eta_{1} \eta_{2}$. The pullback of $\omega$ is

$$
p_{A}^{*} \omega=\frac{h \circ p_{A} \eta_{n+1} d \eta_{1} \wedge \cdots \wedge d \eta_{n+1}}{f \circ p_{A}}=\frac{h \circ p_{A} d \eta_{1} \wedge \cdots \wedge d \eta_{n+1}}{f^{A}} .
$$

Therefore $p_{A}^{*}$ has the proper transform $f^{A}$ as simple polar locus. Doing the same calculation for $p_{B}$, we find $f^{B}=\eta_{1}^{d-1} \eta_{n+1}^{d}-\eta_{2}$ and

$$
p_{B}^{*} \omega=\left(h \circ p_{B} d \eta_{1} \wedge \cdots \wedge d \eta_{n+1}\right) / f^{B},
$$

so that $p_{B}^{*}$ has a pole only along $W^{\prime}$. Note that $f^{B}$ is smooth whereas $f^{A}$ is still singular. However, the ramification of $f^{B}$ has dropped by one. Therefore we

$\left({ }^{15}\right)$ The point is that we blow up along an algebraic subvariety of codimension 2 in $\boldsymbol{P}_{n+1}$. To determine the effect of this globally defined transformation, it is enough to see what happens locally at each point. 
conclude that the process can be repeated $d$ times to obtain a sequence of quadratic transformations

$$
\begin{aligned}
& \boldsymbol{P}_{n+1} \stackrel{p_{1}}{\longleftarrow} Q_{1} \boldsymbol{P}_{n+1} \stackrel{p_{2}}{\longleftarrow} Q_{2} \boldsymbol{P}_{n+1} \stackrel{p_{3}}{\longleftarrow} \ldots \stackrel{p_{d}}{\longleftarrow} Q_{d} \boldsymbol{P}_{n+1} \\
& W \stackrel{p_{1}}{\longleftarrow} W_{1} \stackrel{p_{2}}{\longleftarrow} W_{2} \stackrel{p_{3}}{\longleftarrow} \ldots \stackrel{p_{d}}{\longleftarrow} W_{d}
\end{aligned}
$$

such that at each stage a rational $(n+1)$-form on $Q_{1} \boldsymbol{P}_{n+1}$ with $W_{i}$ as simple polar locus pulls back to give a rational differential form on $Q_{i+1} \boldsymbol{P}_{n+1}$ with a pole only along $W_{i+1}$. In fact, the above diagram is given locally by

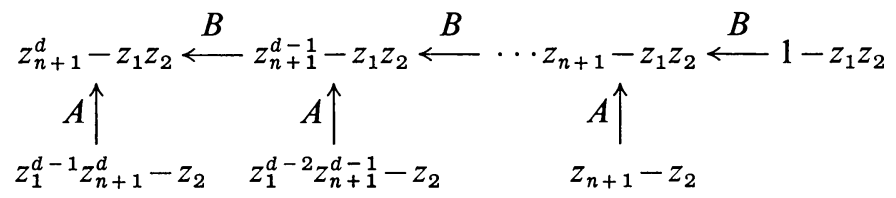

At each stage of the resolution, the computation of the adjoint conditions is the same as above. Moreover, each transform on the second row is a nonsingular piece, whereas the last piece on the first row is nonsingular, so the process terminates with a variety $W_{d}$ having no singularities lying above $\left\{z_{1}=0\right\} \cap\left\{z_{n+1}=0\right\}$ in the original $W$. Applying the same process to the finite number of components in the singular locus yields Lemma 2 in the special case.

Proof of Lemma 2 in general. Suppose that $V_{0}$ is obtained from $\boldsymbol{P}_{n+1}$ by a succession of quadratic transforms with nonsingular centers. Let $W_{0} \subseteq V_{0}$ be the proper transform of $W$, where $W$ is our standard cyclic cover. Let $\Sigma_{0}$ be a nonsingular variety of codimension 2 in $V_{0}$ such that for $x \in \Sigma_{0}, W_{0}$ has the local equation $f=z_{1}^{a} z_{2}^{a}{ }_{2} \cdots z_{l}^{a} z_{n+1}^{a_{n+1}}-z_{l+1} \cdots z_{k}$. Thus $W$ is given by such local equations, where $l=0$. Define the weight of $W_{0}$ along $\Sigma_{0}$ by

$$
\rho\left(W_{0}, \Sigma_{0}\right)=\min \left(k-l, a_{1}+a_{2}+\cdots+a_{l}+a_{n+1}\right) .
$$

Note that if $\rho\left(W_{0}, \Sigma_{0}\right)=1$, then $W_{0}$ is nonsingular along $\Sigma_{0}$. Suppose we can prove that the map $p: V_{1} \rightarrow V_{0}$ obtained by blowing up along $\Sigma_{0}$ satisfies

(a) $p\left(W_{1}, \Sigma_{1}\right)<p\left(W_{0}, \Sigma_{0}\right)$, where $W_{1}, \Sigma_{1}$ are the proper transforms of $W_{0}, \Sigma_{0}$, respectively.

(b) $W_{1}$ is given by local equations as above.

(c) $p_{*}\left(\Omega^{n+1}\left(W_{1}\right)\right)=\Omega^{n+1}\left(W_{0}\right)$, i.e. $p: V_{1} \rightarrow V_{0}$ imposes no adjoint conditions.

Then iteration of this process progressively decreases the weight of the singularities of the transforms $W_{i}$ and no adjoint conditions are imposed at any stage. Applying this to the irreducible components of the singular locus of $W$ establishes the lemma, since the weights all go to zero after a finite number of steps.

To prove the above claim, we consider the local equation $f=z_{1}^{a} z_{2}^{a}{ }_{2} \cdots z_{l}^{a_{l}} z_{n+1}^{a_{n}+1}$ $-z_{l+1} \cdots z_{k}$. Blowing up along $\Sigma_{0}$, we get locally

$$
\begin{aligned}
& f \circ p_{A}=\eta_{n+1}\left(\eta_{1}^{a_{1}} \eta_{2}^{a} \cdots \eta_{l}^{a_{l}} \eta_{n+1}^{a_{n+1}}-\eta_{l+1} \cdots \eta_{k}\right)=\eta_{n+1} f^{A}, \\
& f \circ p_{B}=\eta_{l+1}\left(\eta_{1}^{a_{1}} \eta_{2}^{a_{2}} \cdots \eta_{l}^{a_{l}} \eta_{n+1}^{a_{n+1}-1}-\eta_{l+2} \cdots \eta_{k}\right)=\eta_{l+1} f^{B} .
\end{aligned}
$$


Notice that the proper transforms have the correct form and that in each case, the weight drops by one. If $\omega=\left(h d z_{1} \wedge \cdots \wedge d z_{n+1}\right) / f$ is a rational $(n+1)$-form, we find that

$$
\begin{aligned}
& p_{A}^{*} \omega=\left(h \circ p_{A} d \eta_{1} \wedge \cdots \wedge d \eta_{n+1}\right) / f^{A}, \\
& p_{B}^{*} \omega=\left(h \circ p_{B} d \eta_{1} \wedge \cdots \wedge d \eta_{n+1}\right) / f^{B},
\end{aligned}
$$

so that no adjoint conditions are imposed. This completes the proof of Lemma 2.

Proof of Theorem 1'. We can now show that the desingularization $W^{\prime}$ of the standard cyclic cover $W$ is of general type. For this we use the lemma:

Lemma. Let $g_{K}$ be the map determined by the global sections of $K_{W^{\prime}}$. Suppose that $g_{K}$ has no base points $\left({ }^{16}\right)$ and that $\operatorname{dim}\left(\operatorname{im} g_{K}\right)=\operatorname{dim} W^{\prime}$. Then $W^{\prime}$ is of general type.

Proof. Since $g_{K}$ has no base points, we have a holomorphic map $g_{K}: W^{\prime} \rightarrow W_{1}$ $\subseteq P_{N}$. Here $W_{1}$ is an irreducible, though generally singular variety of dimension $n=\operatorname{dim} W$. By construction, if $H$ is a hyperplane section of $W_{1}$, the $g_{K}^{-1}(H)$ is a divisor belonging to $K_{W^{\prime}}$. Now let $\pi: W_{1} \rightarrow \boldsymbol{P}_{n}$ be a generic linear projection. If $H_{0}$ is a hyperplane in $\boldsymbol{P}_{n}$, then $\pi^{-1}\left(H_{0}\right)$ is a hyperplane section of $W_{1}$. Now

$$
\operatorname{dim} H^{0}\left(P_{n},\left[H_{0}\right]^{k}\right)=\left(\begin{array}{c}
n+k \\
k
\end{array}\right)=O\left(k^{n}\right) .
$$

Therefore, since independent sections of $\left[H_{0}\right]^{k}$ pull back to independent sections of $K_{W}^{k}$, by $\left(\pi \circ g_{K}\right)^{-1}$, we see that $W^{\prime}$ is of general type.

To show that $g_{K}$ has no base points, we must show that there is a section $\phi \in \Gamma\left(K_{W^{\prime}}\right)$ such that $\phi(x) \neq 0$, where $x \in W$ is arbitrary. We first let $x$ be a simple point of $W^{\prime}$. Now the singularities of $W^{\prime}$ are of codimension two, so that we can find a homogeneous polynomial $P_{0}$ of degree $d-(n+2)$ such that $P_{0}(x) \neq 0$. Let

$$
\omega_{0}=\left(P_{0} d z_{1} \wedge \cdots \wedge d z_{n+1}\right) / Q
$$

be the corresponding rational differential form. Since no adjoint conditions are imposed, $R\left(\pi^{*} \omega_{0}\right)$ gives a holomorphic $n$-form on $W^{\prime}$ which does not vanish at $x$. (Recall that outside the exceptional set $\mathscr{E}$, the divisor of $R\left(\pi^{*} \omega_{0}\right)$ is just $\pi^{-1}\left(P_{0}=0\right)$.) Therefore $g_{K}$ has no base points lying above simple points of $W$. Now suppose that $x \in W$ is a singular point. We can perform the same construction, but in general $R\left(\pi^{*} \omega_{0}\right)$ may vanish on $\pi^{-1}(x)$. In our case, however, this does not happen. Referring to the calculation of the adjoint conditions, we see that

$$
\pi^{*}\left(h d z_{1} \wedge \cdots \wedge d z_{n+1}\right) / f=\left(h \circ \pi d z_{1} \wedge \cdots \wedge d z_{n+1}\right) / f^{\pi},
$$

where $f^{\pi}$ is the proper transform of the local defining equation $f$. Therefore, no new zeros are introduced by $\pi^{*}$. Hence $R\left(\pi^{*} \omega_{0}\right) \neq 0$ on $\pi^{-1}(x)$, and so $g_{K}$ is without base points.

${ }^{(16)}$ By this we mean that $g_{K}$ is everywhere defined. If for all $x \in W^{\prime}$ there is a section $\phi \in \Gamma\left(K_{W^{\prime}}\right)$ such that $\phi(X) \neq 0$, then $g_{K}$ has no base points. 
To see that $\operatorname{dim}\left(\operatorname{im} g_{K}\right)=\operatorname{dim} W^{\prime}$, we pick a simple point $x \in W$, and select forms of degree $d-(n+2), P_{0}, \ldots, P_{n}$, such that $P_{0}(x) \neq 0$ and the divisors $\left(P_{i}=0\right)$ meet transversely at $x$ for $i=1, \ldots, n$. We can do this because $[H]^{d-(n+2)}$ is very ample. Now let $\phi_{i}=R\left(\pi^{*} \omega_{i}\right)$, where $\omega_{i}$ is the rational differential determined by $P_{i}$. Among the coordinate functions of $g_{K}$ are $\phi_{1} / \phi_{0}, \ldots, \phi_{n} / \phi_{0}$. The fact that the divisors $\left(P_{i}=0\right)$ meet transversely at $x$ shows that the $\phi_{i} / \phi_{0}$ give local coordinates there. Therefore the Jacobian of $g_{K}$ has maximal rank at $x$, proving the claim. This completes the proof of Theorem $1^{\prime}$.

Remarks. (1) One can compute the dimension of $H^{\circ}\left(W^{\prime}, K_{W^{\prime}}^{m}\right)$ explicitly to show that $W$ is of general type. The proof is not hard and is essentially contained in the calculation of the adjoint conditions.

(2) The fact that we can find a polynomial $P$ of degree $d-(n+2)$ avoiding the singularities of $W$ shows that there is a holomorphic $n$-form $\phi$ on $W^{\prime}$ which does not meet $\mathscr{E}$. Therefore the canonical bundle $K_{W}$, restricted to any component of $\mathscr{E}$ is trivial. This shows that $\mathscr{E}$ is collapsed by all the pluricanonical maps $g_{K^{m}}$.

\section{Miscellaneous remarks and examples.}

(1) The classical little Picard theorem can be obtained by the above techniques. To see this, let $W$ be a five-sheeted branched cover of $\boldsymbol{P}_{1}$, branched with ramification index 4 at 0,1 , and $\infty$. Such a Riemann surface is guaranteed by the Riemann existence theorem $\left({ }^{17}\right)$. Alternatively, one may desingularize the algebraic curve determined by the affine equation $z^{5}=x(x-1)$. If $\rho$ is the total ramification index of $W$, the Hurwitz formula gives $\rho=2(g(W)-n+1)$, where $n$ is the number of sheets. Hence we find $g(W)=2$. From Riemann surface theory we know that $K_{W}^{3}$ is very ample. Now let $f: \boldsymbol{C} \rightarrow \boldsymbol{P}_{1}-\{0,1, \infty\}$. We lift to $f^{\prime}: \boldsymbol{C} \rightarrow W$, and apply the slightly strengthened version of Griffiths' canonical algebraic varieties theorem to conclude that $f^{\prime}$, and hence $f$, is constant.

We remark that this method fails to show that $f: \boldsymbol{C}^{n} \rightarrow \boldsymbol{P}_{n}-\{n+2$ hyperplanes in general position $\}$ is totally degenerate.

(2) As another example, we prove the classical theorem that any holomorphic $f: C \rightarrow \mathscr{E}-x$ is constant, where $\mathscr{E}$ is an elliptic curve and $x \in \mathscr{E}$ is an arbitrary point. Let $\mathscr{E}_{1} \stackrel{\pi_{1}}{\longrightarrow} \mathscr{E}$ be a two-sheeted unramified cover of $\mathscr{E}$. By the Hurwitz formula, $\mathscr{E}_{1}$ is an elliptic curve. Now let $W \stackrel{\pi_{2}}{\longrightarrow} \mathscr{E}_{1}$ be a two-sheeted branched cover of $\mathscr{E}_{1}$ branched at $\left\{y_{1}, y_{2}\right\}=\pi^{-1}(x)\left({ }^{18}\right)$. Now the Hurwitz formula $\rho=2\left(g^{\prime}-n g+n-1\right)$

${ }^{\left({ }^{17}\right)}$ This says that a Riemann surface can be constructed by knowing how the sheets interchange around the branch points. To be explicit, let $A_{1}, \ldots, A_{k}$ generate a transitive permutation group on $\{1, \ldots, n\}$. Suppose that $A_{1} \cdot A_{2} \cdots A_{k}=1$. Then there is a Riemann surface $W$ which is an $n$-sheeted branched cover of $\boldsymbol{P}_{1}$, branched at points $x_{1}, \ldots, x_{n}$. The effect of going around $x_{i}$ is described by $A_{i}$.

${ }^{(18)}$ In general, if $V$ is a complex manifold, $D \subseteq V$ a smooth divisor, there is a $k$-sheeted cyclic cover of $V$ branched along $D$ if and only if $c_{1}([D])$ is divisible, i.e. there is an integral cohomology class $\beta$ such that $k \beta=c_{1}([D])$. (See J. Wavrick, Deformations of branched coverings of complex manifolds, Amer. J. Math. 90 (1968), 929.) 
shows that $g^{\prime}=g(W)=2$. By lifting $f$ from $\mathscr{E}-x$ to $\mathscr{E}_{1}-\left\{y_{1}, y_{2}\right\}$ and then to $W_{1}$ and then applying Griffiths' theorem to $W$, we see that $f$ must be constant.

REMARK. Using the same trick for "multiplying the Chern class of $D$ " we may show that if $A$ is an abelian variety and $D$ is a smooth divisor such that the intersection number $\left(D^{n}\right)>0$, then any holomorphic map $f: C^{n} \rightarrow A-D$ is totally degenerate.

(3) We can prove the following generalization of the big Picard theorem:

Proposition. Let $V$ be a nonsingular hypersurface of degree $d \geqq n+3$ in $\boldsymbol{P}_{n}$. Let $f$ be a nondegenerate holomorphic map $f: \Delta^{*} \times \Delta^{n-1} \rightarrow \boldsymbol{P}_{n}-V$. Then $f$ extends to a holomorphic map $f: \Delta^{n} \rightarrow \boldsymbol{P}_{n}$.

Proof. Let $W$ be the usual canonical algebraic variety which is a $d$-sheeted cyclic cover of $\boldsymbol{P}_{n}$ branched along $V$. Consider the diagram

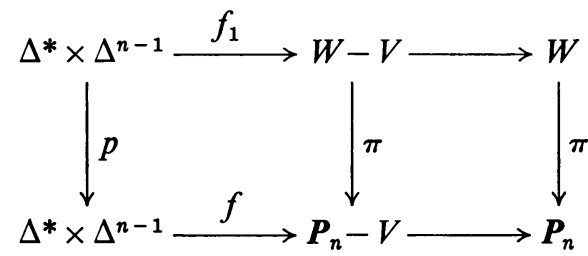

where $p\left(z_{1}, \ldots, z_{n}\right)=\left(z_{1}^{d}, z_{2}, \ldots, z_{n}\right)$ and $f_{1}$ is a lifting of $f$. By Griffiths' theorem, $f_{1}$ extends to a holomorphic map $\bar{f}_{1}: \Delta^{n} \rightarrow W$. This implies (by commutativity of the diagram) that $f$ is locally bounded. Thus $f$ extends to $\bar{f}$ by the Riemann extension theorem.

REMARK. The classical big Picard theorem can also be proved by these methods. In the argument above we let $W$ be the five-sheeted cyclic cover constructed in (1). Since $K_{W}^{3}$ is very ample, we know that the lifting of $f: \Delta^{*} \rightarrow \boldsymbol{P}_{1}-\{0,1, \infty\}$ to $f^{\prime}: \Delta^{*} \rightarrow W$ extends as a rational map. Now for one complex variable, there are no points of indeterminacy, so $f^{\prime}$ is holomorphic. The argument now proceeds as before.

The above theorem has several useful corollaries.

COROllaRy 1. Let $f: A \rightarrow \boldsymbol{P}_{n}-V$ be holomorphic, where $A$ is an open algebraic variety and $V$ is smooth and of degree $d \geqq n+3$. Then $f$ extends to a holomorphic map $\bar{f}: \bar{A} \rightarrow \boldsymbol{P}_{n}$, for any smooth compactification $\bar{A}$.

Proof. Immediate, using the above proposition. Any smooth compactification works because the local extensions are holomorphic, so we do not have to worry about points of indeterminacy.

COROllaRy 2. Let $V_{1}, V_{2}$ be two smooth hypersurfaces of degree $d \geqq n+3$ in $\boldsymbol{P}_{n}$. Then $\boldsymbol{P}_{n}-V_{1}$ is biholomorphic to $\boldsymbol{P}_{n}-V_{2}$ if and only if there is a projective linear automorphism of $\boldsymbol{P}_{n}$ which carries $V_{1}$ onto $V_{2}$. 
Proof. Let $f: \boldsymbol{P}_{n}-V_{1} \rightarrow \boldsymbol{P}_{n}-V_{2}$ and $g: \boldsymbol{P}_{n}-V_{2} \rightarrow \boldsymbol{P}_{n}-V_{1}$ be maps exhibiting the isomorphism. Thus $f \circ g=\mathrm{id}$ and $g \circ f=\mathrm{id}$. By Corollary $1, f$ extends to a holomorphic map $\bar{f}: \boldsymbol{P}_{n} \rightarrow \boldsymbol{P}_{n}$, and we obviously have $\bar{f}\left(V_{1}\right)=V_{2}$. We have a similar extension $\bar{g}$ of $g$. Now $\bar{g} \circ \bar{f}$ restricts to the identity map on the open set $\boldsymbol{P}_{n}-V_{1}$, so $\bar{g} \circ \bar{f}=\operatorname{id}_{\boldsymbol{P}_{n}}$. The same reasoning shows that $\bar{f} \circ \bar{g}=\operatorname{id}_{\boldsymbol{P}_{n}}$, hence $\bar{f}$ is a biholomorphic map of $\boldsymbol{P}_{n}$ into itself. Now the only holomorphic automorphisms of $\boldsymbol{P}_{n}$ are the projective linear automorphisms, which completes the proof.

Corollary 3. The automorphism group of $\boldsymbol{P}_{n}-V$ is finite.

Proof. Any automorphism $\beta$ of $\boldsymbol{P}_{n}-V$ lifts to an automorphism of $W-V$, where $W$ is the standard $d$-fold cyclic cover of $\boldsymbol{P}_{n}$ branched along $V$. The same argument as in the proof of Corollary 2 shows that $\beta$ extends to an automorphism $\bar{\beta}$ of $W$. Now the automorphism group of a canonical algebraic variety is always finite (see [7]).

(4) We close by noting that there are many particular cases in which very strong degeneracy theorems can be obtained by essentially one-variable techniques. For example, let $|H(d)|$ be the linear system of hypersurfaces of degree $d$ on $\boldsymbol{P}_{n}$. Pick $f_{0}, f_{1} \in|H(d)|$ to be independent. Then the set of hypersurfaces $\left\{\lambda f_{0}+\mu f_{1}\right\}$ forms a pencil. We set $f(\alpha)=f_{0}+\alpha f_{1}$ and $f(\infty)=f_{0}$. Notice that all the $f(\alpha)$ intersect along the base locus $B=f(0) \cap f(\infty)$. Now the pencil $\mathscr{P}$ gives a holomorphic map $\pi: \boldsymbol{P}_{n}-\{f(0), f(1), f(\infty)\} \rightarrow \boldsymbol{P}_{1}-\{0,1, \infty\}$ by sending $x \rightarrow\left[f_{0}(x), f_{1}(x)\right]$. If $g: \boldsymbol{C}$ $\rightarrow \boldsymbol{P}_{n}-\{f(0), f(1), f(\infty)\}$ is holomorphic, we conclude by Picard's theorem applied to $\pi \circ g$ that the image of $g$ lies in some fiber $f(\alpha)$. By applying this argument to all complex lines through the origin in $\boldsymbol{C}^{m}$, we see that the image of $g: \boldsymbol{C}^{m} \rightarrow \boldsymbol{P}_{n}$ $-\{f(0), f(1), f(\infty)\}$ lies in a fiber.

Using this observation, we see that by using several pencils in general position we can require im $(g)$ to lie in the transverse intersection of several fibers. In this way we can progressively decrease the dimension of im $(g)$ and in all cases im $(g)$ lies in an algebraic subvariety. We can also drop the dimension of im $(g)$ by increasing the degree of the pencil. For example, let $\mathscr{P}$ be a pencil of generically nonsingular cubic curves in $\boldsymbol{P}_{2}$. There will be a certain number of singular fibers $C\left(\alpha_{1}\right), \ldots, C\left(\alpha_{k}\right)$. If $k<3$ we add more nonsingular fibers to get $k \geqq 3$. Now let $f: \boldsymbol{C} \rightarrow \boldsymbol{P}_{2}-\left(C\left(\alpha_{1}\right) \cdots C\left(\alpha_{k}\right)\right)$. Then im $(f)$ lies in $C(\alpha)-C\left(\alpha_{1}\right)$, where $\alpha=\alpha_{i}$, $i=1, \ldots, k$. In other words, $f$ maps into an elliptic curve minus at least one point, and hence is constant.

REMARKS. (1) The above examples are not essentially deep, since they use only one complex variable techniques. However, they lead one to ask whether there are conditions on a divisor $D \subseteq P_{n}$ such that for $f: \boldsymbol{C}^{n-1} \rightarrow \boldsymbol{P}_{n}-D$, im $(f)$ lies in a divisor, preferably a divisor in the complete linear system determined by $D$.

(2) The above examples show how the requirement of general position makes things harder. In fact, if we look at a configuration of more than three lines in $\boldsymbol{P}_{2}$, then either they are in general position or they contain three elements of a pencil. 


\section{BIBLIOGRAPHY}

1. S. S. Chern, Differential geometry, its past and future, Internat. Congress Math., Nice, France, 1970.

2. P. Griffiths, Hermitian differential geometry, Chern classes, and positive vector bundles, Global Analysis (Papers in Honor of K. Kodaira), Univ. Tokyo Press, Tokyo, 1969, pp. 185251. MR 41 \#2717.

3. - Holomorphic mappings into canonical algebraic varieties, Ann. of Math. 93 (1971), $439-458$.

4. - - On the periods of certain rational integrals. I, II, Ann. of Math. (2) 90 (1969), 460-495; ibid., 496-541. MR 41 \#5357.

5. H. Grauert, Über Modifikationen und exzeptionelle analytische Mengen, Math. Ann. 146 (1962), 331-368. MR 25 \#583.

6. F. Hirzebruch, Topological methods in algebraic geometry, 2nd ed., Ergebnisse der Mathematik und ihrer Grenzgebiete, Heft 9, Springer-Verlag, Berlin, 1962; English transl., Die Grundlehren der math. Wissenschaften, Band 131, Springer-Verlag, New York, 1966. MR 25 \#1155; MR 34 \#2573.

7. S. Kobayashi, On the automorphism group of a certain class of algebraic manifolds, Tôhoku Math. J. (2) 11 (1959), 184-190. MR 22 \#3014.

8. K. Kodaira, On holomorphic mappings of polydiscs into compact complex manifolds (to appear).

9. P. Lelong, Plurisubharmonic functions and differential forms, Gordon and Breach, New York, 1969.

10. W. Rudin, Real and complex analysis, McGraw-Hill, New York, 1966. MR 35 \#1420.

11. H. Wu, The equidistribution theory of holomorphic curves, Ann. of Math. Studies, no. 64, Princeton Univ. Press, Princeton, N. J., 1970.

Department of Mathematics, Princeton University, Princeton, New Jersey 08540

Current address: Department of Mathematics, Stanford University, Stanford, California 94305 